\title{
Eosinophils are an integral component of the pulmonary granulocyte response in Tuberculosis and promote host resistance in mice
}

\author{
Andrea C. Bohrer ${ }^{1,19}$, Ehydel Castro ${ }^{1,19}$, Zhidong Hu ${ }^{2,3,19}$, Artur T.L. Queiroz ${ }^{4}$, Claire E. Tocheny \\ 1, Maike Assmann ${ }^{1}$, Shunsuke Sakai ${ }^{5}$, Christine Nelson ${ }^{5}$, Paul J. Baker ${ }^{1}$, Hui Ma ${ }^{2,3}$, Lin Wang \\ ${ }^{3,6}$, Wen Zilu ${ }^{3,6}$, Elsa du Bruyn ${ }^{7}$, Catherine Riou ${ }^{7}$, Keith D. Kauffman ${ }^{5}$, Tuberculosis Imaging \\ Program ${ }^{8}$, Ian N. Moore ${ }^{9}$, Franca Del Nonno ${ }^{10}$, Linda Petrone ${ }^{11}$, Delia Goletti ${ }^{11}$, Adrian R. \\ Martineau ${ }^{12}$, David M. Lowe ${ }^{12}$, Mark R. Cronan ${ }^{13,14}$, Robert J. Wilkinson ${ }^{7,15,16}$, Clifton E. Barry \\ III ${ }^{7,17}$, Laura E. Via ${ }^{8,17}$, Daniel L. Barber ${ }^{5}$, Amy D. Klion ${ }^{18}$, Bruno B. Andrade ${ }^{4}$, Yanzheng Song \\ ${ }^{3,6}$, Ka-Wing Wong ${ }^{2,3,20}$ and Katrin D. Mayer-Barber 1,20,21
}

1 Inflammation and Innate Immunity Unit, Laboratory of Clinical Immunology and Microbiology, National Institute of Allergy and Infectious Diseases (NIAID), National Institutes of Health (NIH), Bethesda, 20892, USA.

2 Department of Scientific Research, Shanghai Public Health Clinical Center (SPHCC), Fudan University, Shanghai, 201508, China.

3 TB Center, Shanghai Emerging and Re-emerging Infectious Disease Institute, Fudan University, Shanghai, 201508, China.

4 The KAB group, Multinational Organization Network Sponsoring Translational and Epidemiological Research (MONSTER) Initiative, Instituto Gonçalo Moniz, Fundação Oswaldo Cruz, Salvador 40296-710, Brazil 5 T Lymphocyte Biology Section, LPD, NIAID, NIH, Bethesda, 20892, USA.

6 Department of Thoracic Surgery, Shanghai Public Health Clinical Center (SPHCC), Fudan University, Shanghai 201508, China.

7 Wellcome Centre for Infectious Diseases Research in Africa, Institute of Infectious Disease and Molecular Medicine, University of Cape Town, Observatory, 7925, South Africa.

8 Tuberculosis Imaging Program (TBIP), DIR, NIAID, NIH, Bethesda, 20892, USA.

9 Infectious Disease Pathogenesis Section, Comparative Medicine Branch, NIAID, NIH, Bethesda, 20892, USA.

10 Pathology Unit, National Institute for Infectious Diseases "L. Spallanzani", IRCCS, Rome, Italy.

11 Translational Research Unit, Department of Epidemiology and Preclinical Research National Institute for Infectious Diseases-IRCCS, Roma, 00149, Italy. 
12 Institute of Immunity and Transplantation, University College London, Royal Free Campus, Pond Street, London, NW3 2QG, UK.

13 In Vivo Cell Biology of Infection Unit, Max Planck Institute for Infection Biology, 10117 Berlin, Germany.

14 Department of Molecular Genetics and Microbiology, Duke University School of Medicine, Durham, 27710, USA.

15 Department of Infectious Diseases, Imperial College London, W12 ONN, UK

16 Francis Crick Institute, Midland Road, London, NW1 1AT, UK.

17 Tuberculosis Research Section, LCIM, NIAID, NIH, Bethesda, 20892, USA.

18 Human Eosinophil Section, Laboratory of Parasitic Diseases (LPD), NIAID, NIH, Bethesda 20812, USA.

19 These authors contributed equally

20 Co-Corresponding authors

21 Corresponding author and lead contact

* mayerk@niaid.nih.gov

\section{ABSTRACT}

Host resistance to Mycobacterium tuberculosis infection requires the activities of multiple leukocyte subsets, yet the roles of the different innate effector cells during tuberculosis are incompletely understood. Here we uncover an unexpected association between eosinophils and Mtb infection. In humans, eosinophils are decreased in the blood but enriched in resected human tuberculosis lung lesions and autopsy granulomas. Influx of eosinophils is also evident in infected zebrafish, mice, and nonhuman primate granulomas, where they are functionally activated and degranulate. Importantly, employing complementary genetic models of eosinophil deficiency, we demonstrate that, in mice, eosinophils are required for optimal pulmonary bacterial control and host survival after Mtb infection. Collectively, our findings uncover an unexpected recruitment of eosinophils to the infected lung tissue and a protective role for these cells in the control of Mtb infection in mice. 


\section{KEYWORDS:}

Mycobacterium tuberculosis; lung; bacterial infection; granulocytes, eosinophils, eosinophil peroxidase, nonhuman primate, macaque

\section{INTRODUCTION}

Mycobacterium tuberculosis $(M t b)$ is an intracellular bacterium that causes tuberculosis (TB), which through 2020 accounted for the highest yearly global mortality due to a single pathogen (WHO, 2020). Mtb primarily infects innate immune cells and host resistance depends on anti-microbial effector functions of infected macrophages alongside a robust type I and T helper 1 immune response (Cooper et al., 1993; Ernst, 2012; Flynn et al., 1993; Flynn et al., 2015). Eosinophils, in contrast, are major innate effector cells in type II inflammatory settings (Klion and Nutman, 2004; Simon et al., 2020). Accordingly, reports of type II immunity-associated cells and responses during Mtb infection are rare with one report of eosinophils in lungs of $M t b-$ infected guinea pigs (Lasco et al., 2004) and one case series of pulmonary eosinophilia in three TB patients (Vijayan et al., 1992). As a result, eosinophils have been assumed to be largely uninvolved in the immune response to Mtb or actively repressed by the strong IFNY-driven responses required for control of mycobacteria (Cooper et al., 1993; Flynn and Ernst, 2000; Kirman et al., 2000; O'Garra et al., 2013).

Studies of eosinophil responses in vivo have historically focused on allergic responses and parasitic helminth infections (Klion et al., 2020; Travers and Rothenberg, 2015). Eosinophils are associated with an established type II response during lung infections with respiratory syncytial virus and Aspergillus (Lilly et al., 2014; Phipps et al., 2007) and recent data highlight complex roles for eosinophils in tissue homeostasis and remodeling, barrier function and wound healing (Lee et al., 2010; Rosenberg et al., 2013; Shah et al., 2020). Indeed, eosinophils are pluripotent, armed with a variety of bioactive molecules stored preformed in granules or synthesized in lipid bodies. These include a large repertoire of cytokines, chemokines, lipid 
mediators and cationic granule proteins including eosinophil cationic protein, eosinophil-derived neurotoxin, eosinophil peroxidase (EPX) and major basic protein (Acharya and Ackerman, 2014; Klion et al., 2020; Shamri et al., 2011). However, our understanding of the in vivo function of eosinophils during bacterial infections is limited. Reports are currently restricted to extracellular bacteria where direct cell-autonomous bactericidal properties of eosinophils, including extracellular DNA traps and bactericidal granule proteins, can promote extracellular clearance of Escherichia coli, Citrobacter rodentium, Staphylococcus aureus and Pseudomonas aeruginosa bacteria (Arnold et al., 2018; Krishack et al., 2019; Linch et al., 2009; Yousefi et al., 2008). However, the physiological role of eosinophils in host resistance to intracellular bacterial infections remains unknown.

Here we establish that eosinophils are a significant cellular component of TB granulomas and Mtb-infected lungs in mice, nonhuman primates (NHP) and patients. Using complementary genetic approaches to create eosinophil deficiency, we demonstrate that eosinophil responses are required for optimal host resistance and survival after $M$ tb infection in mice. Together our findings reveal a previously unrecognized association between eosinophils and TB in multiple hosts and uncover a protective role for these cells in mice.

\section{RESULTS AND DISCUSSION}

We first investigated whether eosinophils may participate in the granulocytic response to TB by quantifying circulating eosinophils in three independent clinical cohort studies (Du Bruyn et al., 2020; Lowe et al., 2013; Mayer-Barber et al., 2014). We asked whether the numbers of peripheral blood eosinophils of TB patients changed based on disease status and antitubercular treatment (ATT) in a South African cohort (Du Bruyn et al., 2020). Indeed, blood eosinophil numbers were significantly higher in individuals with latent infection (LTBI) compared to active pulmonary TB (PTB) patients (Fig. 1 A), with a corresponding decreased eosinophil to neutrophil (E/N ratio) in the PTB group (Fig. S1 A-B). When patients in an independent Chinese cohort (Mayer-Barber et 
al., 2014) were stratified according to disease severity based on sputum positivity for acid-fast staining of bacilli (AFB), individuals with active AFB+ PTB and extrapulmonary TB (EPTB) disease exhibited a significantly decreased E/N ratio compared to AFB- PTB patients (Fig. S1 C). These data suggest that a decrease in circulating $\mathrm{E} / \mathrm{N}$ ratio may reflect an increase in sputum bacterial loads. Consistent with the hypothesis that blood eosinophil numbers reflect bacterial sputum status and disease burden, circulating blood eosinophil numbers increased significantly within two weeks of commencing ATT in both cohorts (Fig.1 B, Fig. S1 D). Moreover, in a third British cohort that included patients with both PTB and EPTB (Lowe et al., 2013), TB disease survivors exhibited a significantly higher $\mathrm{E} / \mathrm{N}$ ratio on blood tests taken at the time of diagnosis, compared to individuals who subsequently died (Fig. S1 E). In fact, lower baseline eosinophil numbers in blood correlated with shorter time to TB related death (Fig. S1 F). Taken together, our data from three independent clinical cohorts suggest that peripheral blood eosinophils are decreased according to disease severity, raising the possibility that rather than being depleted, eosinophils may be recruited to distant disease sites where they could participate in the local immune response to infection.

To directly address whether eosinophils could be part of the local pulmonary granulocyte response in human TB we measured the relative abundance of granulocytes in human TB lesions. To this end we generated single cell suspensions from freshly resected human lung tissue $(n=9$ TB patients) directly after clinically indicated surgery and identified tissue-resident granulocytes with established markers Siglec-8 ${ }^{+} / \mathrm{CD} 66 \mathrm{~b}^{+}$for eosinophils and Siglec-8 ${ }^{\text {neg }} / \mathrm{CD} 66 \mathrm{~b}^{+}$for neutrophils (Fig. 1 C, Fig. S1 G). Neutrophils were abundant CD45+ immune cells in human lung lesions divided into different macro-pathologies (Fig. S1 H). However, eosinophils were also frequently present in appreciable numbers relative to neutrophils in the same lung lesion sample (Fig. 1 D, Fig. $\mathbf{S 1 ~ H ) . ~ I n ~ f a c t , ~ E / N ~ r a t i o s ~ a n d ~ e o s i n o p h i l ~ f r e q u e n c i e s ~ o f ~} \mathrm{CD} 45^{+}$immune cells were sometimes similar to or even higher than neutrophils in some, but not all, samples from fibrotic areas and granulomas compared to the blood in the same individuals (Fig. 1 D-E, Fig. S1 H-I). We then 
asked whether eosinophils would selectively infiltrate metabolically active inflamed lung lesions via 2-deoxy-2-fluorine-18-fluoro-D-glucose ( $\left.{ }^{18} \mathrm{FDG}\right)$ positron emission tomography/computed tomography (PET/CT) since the more recent patients received ${ }^{18} \mathrm{FDG}$ PET/CT scans to aid in defining the surgical regions prior to resection. When we separated individual lesions based on high or low metabolic activity via ${ }^{18}$ FDG signal intensity, eosinophils were significantly increased relative to neutrophils in PET high compared to PET low lung lesions (Fig. 1 F, Fig. S1 J). These data suggest that eosinophils are more abundant in PET FDG-avid areas compared to areas of low metabolic activity and disease. While larger studies are needed to accurately categorize and associate the abundance of specific granulocyte subsets within diverse TB lesion types, our small retrospective study showed that eosinophils can be substantially enriched in individual human TB lesions. Most importantly, the $\mathrm{E} / \mathrm{N}$ ratio was significantly higher in resected human lung lesion types compared to circulation in the same patients (Fig. $\mathbf{1 ~ G ) , ~ s u p p o r t i n g ~ o u r ~ h y p o t h e s i s ~ t h a t ~}$ peripheral blood eosinophils decrease during active TB disease and enrich at infected tissue sites. While a functional role for eosinophils in human disease remains unclear, our clinical data support the hypothesis that during TB, circulating peripheral blood eosinophils are dynamically regulated based on disease status and that eosinophils can be enriched at the Mtb-infected local tissue site.

To confirm the flow cytometric data, we next examined human TB granuloma sections from an independent autopsy-cohort (Blauenfeldt et al., 2018) (Fig. 2 A). Hematoxylin and eosin (H\&E) staining indicated that eosinophils were present primarily in the rim area of the granulomas (Fig. S2 A), whereas diffuse staining for the eosinophil granule protein EPX was observed in the necrotic core of granulomas, suggesting eosinophil degranulation in the center of TB granulomas (Fig. 2 A, Fig. S2 A). To experimentally explore the role of eosinophils, we employed a NHP model in which Mtb infection forms bona-fide lung granuloma structures closely resembling those found in humans (Basaraba and Hunter, 2017; Flynn et al., 2015). We first assessed lung granulomas from Mtb-infected macaques at 7-12 weeks post infection for eosinophil infiltration (Fig. S2 B). Like the human TB granulomas, eosinophils were evident in the outer rim area in 
addition to more diffuse EPX staining in the necrotic core of some granulomas, suggestive of degranulated eosinophils (Fig. 2 B, Fig. S2 C-D). We then asked whether eosinophil recruitment to mycobacterial granulomas was an evolutionarily conserved process. Using the zebrafish model, we assessed eosinophils in mycobacterial granulomas formed after $M$. marinum infection (Parikka et al., 2012; Swaim et al., 2006). Indeed, in agreement with our findings in NHP and human TB granulomas, eosinophils were also found in necrotic granulomas in $M$. marinum infected zebrafish (Fig. 2 C). Taken together, our data show that eosinophils are present in mycobacterial granulomas in human, NHP and zebrafish, suggesting that infiltration of eosinophils into granulomas may be a conserved response to mycobacteria across species.

To measure eosinophil activation and infiltration into granulomas we examined NHP granulomas in greater detail. In order to quantify and functionally assess eosinophils at the single cell level in rhesus macaques, we developed a flow cytometric approach based on EPX (Fig. S2 E). We found that intracellular staining with EPX selectively and specifically stained eosinophils in whole blood (WB), as well as in bronchoalveolar lavage fluid (BAL) (Fig. 3 A). Importantly, when we quantified eosinophils in NHP TB granulomas, we observed a high level of heterogeneity between individual granulomas with eosinophils spanning four orders of magnitude, comprising up to $10 \%$ of all CD45+ immune cells with an average of $1.5 \%$ in 46 granulomas from seven animals (Fig. 3 B, Fig. S2 F). There was no correlation between individual granuloma bacterial loads and eosinophils abundance (Fig. 3 C). Since, diffuse EPX staining in the core of human and NHP granulomas was suggestive of functional activation resulting in degranulation, we next quantified eosinophil degranulation by measuring CD63 surface expression (Fig. 3 D). While blood eosinophils showed little change in CD63 expression, BAL and granuloma eosinophils exhibited significantly increased CD63 expression with the highest CD63 expression in granulomas (Fig. 3 D). The proportion of $\mathrm{CD} 63^{+}$eosinophils varied widely between individual granulomas even within the same animal and was strongly negatively correlated with the abundance of eosinophils (Fig. 3 E), supporting the idea that eosinophil degranulation precedes 
cell death. Importantly, CD63 expression on eosinophils inversely correlated with bacterial burden in granulomas (Fig. 3 F), suggesting that functional activation of eosinophils in form of degranulation may impact bacterial growth in Mtb lesions. Collectively, our data from Mtb infected NHP lesions reveal a high degree of heterogeneity in the eosinophilic response and support the hypothesis that eosinophils migrate into TB granulomas where they degranulate and participate in the local innate immune response against $M t b$.

As a next step in examining the function of eosinophils during Mtb infection we quantified eosinophils after low dose aerosol Mtb infection in the genetically tractable C57BL6 (B6) mouse model. We measured lung tissue resident eosinophils by flow cytometry using an intravenous (i.v.) labelling technique that distinguishes between cells located in the lung vascular capillary bed (i.v. ${ }^{\text {pos}}$ ) and cells that migrated into the lung tissue parenchyma (i.v. ${ }^{\text {neg}}$ ) (Anderson et al., 2014). When we quantified the number of lung tissue resident i.v. ${ }^{\text {neg }}$ eosinophils after $M t b$ infection, we observed an increase after two-three months of Mtb infection compared to either uninfected animals or 30 days post infection (dpi) (Fig. 4 A, Fig. S3 A-B). To ask whether eosinophils could be directly harboring $M t b$, either by infection or phagocytosis, we assessed bacteria-containing eosinophils using fluorescent Mtb-mCherry and found that eosinophils are not infected with Mtb (Fig. 4 B). In fact, Mtb-infected cells have been identified and described in detail (Cohen et al., 2018; Huang et al., 2018; Lee et al., 2020; Pisu et al., 2020; Rothchild et al., 2019; Wolf et al., 2007), and we like others have consistently failed to detect significant numbers of Mtb-containing eosinophils (Lee et al., 2020; Rothchild et al., 2019). Thus, while eosinophils may interact with Mtb or Mtb-infected cells in lungs and TB granulomas, they themselves do not appear to represent a cellular niche for Mtb. Based on the increase in lung parenchymal eosinophils after infection and their role in tissue homeostasis (Lee et al., 2010; Rosenberg et al., 2013; Shah et al., 2020), we asked whether the absence of eosinophils after Mtb infection would lead to noticeable changes in lung pathology. To this end we infected B6 $\Delta \mathrm{db}$ IGata mice, that lack eosinophils due to a targeted deletion of a high-affinity GATA-binding site in the GATA-1 promoter (Yu et al., 2002). 
However, we did not observe changes in lung gross pathology three months after Mtb infection in B6 $\Delta$ dblGata compared to wildtype (WT) mice (Fig. 4 C). We then examined lung resident immune cells and found no changes in i.v.neg Mtb-specific CD4 ${ }^{+}$and CD8 ${ }^{+}$T cells (Fig. S3 C-F), NK1.1 ${ }^{+}$ cells (Fig. S3 G), neutrophils (Fig. S3 H), interstitial macrophage/dendritic cell (DC2) polarization states (Fig. S3 I), alveolar macrophages (Fig. S3 J) or XCR1+ DC1 (Fig. S3 K) in B6 $\Delta$ dbIGata mice compared to WT mice three months after Mtb infection. Lastly, to explore the impact of eosinophil deficiency in an unbiased fashion, we performed whole lung transcriptional profiling on three-month Mtb infected B6 $\Delta \mathrm{db}$ IGata or WT mice alongside uninfected controls (d0). Relatively few genes were significantly and differentially expressed when we directly compared transcripts from d90pi B6 $\Delta$ dblGata and d90pi WT mouse lungs. 16 differentially expressed genes (DEG) were up-regulated and nine down-regulated in $M t b$ infected $\Delta \mathrm{dblGata}$ lungs compared to $M t b$ infected B6 control lungs (Fig. S3 L). Intriguingly, most of these DEG were not associated with immunity to infection, but instead linked to neurological disorders and neuronal pathways (Fig. S3 L, pink annotation). To explore infection induced changes we analyzed and compared d90pi B6 $\Delta$ dbIGata and d90pi WT mouse lungs to d0 WT controls. We identified 194 up- and 262 downregulated DEG in Mtb infected $\triangle \mathrm{dblGata}$ lungs, alongside 94 up- and 230 down-regulated DEG in Mtb infected WT B6 lungs (Fig. S3 M). The top 20 DEG were again enriched in neuronal-, sensory- and olfactory-associated genes (pink annotation) in addition to genes related to lipid metabolism (green annotation) (Fig. 4 D). Consistent with limited changes in genes linked to host resistance, transcriptional module exploration for type I and type II interferons (Singhania et al., 2019), confirmed the restricted molecular perturbations in Mtb infected eosinophil deficient compared to WT lungs (Supp. xls file). Importantly, gene set enrichment analyses revealed again downregulation of pathways associated with neuronal processes, neurological disorders and short chain fatty acid, endocannabinoid, and arachidonic acid metabolism in the lungs of Mtbinfected $\Delta$ dblGata mice (Fig. 4E, Supp. xls file). Eosinophils produce a variety of bioactive arachidonic acid derived lipid mediators some of which have been implicated in host-resistance 
to Mtb infection before (Mayer-Barber and Sher, 2015). Furthermore, endocannabinoids and arachidonic acid derivatives interact with sensory neurons in the airways (Bozkurt, 2019; Chesne et al., 2019). One possible interpretation of the neuronal-associated transcriptional changes could be that they reflect alterations in airway sensory neurons or pulmonary neuroendocrine cells (PNEC) of the lung epithelium. Thus, while our transcriptional profiling of Mtb infected lungs revealed neuronal-associated pathways as being primarily affected by the lack of eosinophils, the biological significance of these transcriptional changes remains unknown. Moreover, the pulmonary-neuronal axis in host resistance against Mtb infection is largely unexplored yet there is growing appreciation for the importance of the cross talk between the nervous and immune system (Veiga-Fernandes and Mucida, 2016). For instance, a recent study found that Mtb-derived sulfolipids can directly bind and activate nociceptive neurons in the lung (Ruhl et al., 2020). Eosinophils have been further shown to alter parasympathetic nerve function and airway sensory nerve density thereby affecting reflex bronchoconstriction in asthma (Costello et al., 1997; Drake et al., 2018a; Drake et al., 2018b; Fryer and Wills-Karp, 1991; Kingham et al., 2002). Thus, our findings raise the possibility that eosinophils may be involved in pulmonary tissue-immune-nerve cross talk during chronic infections.

Finally, to test whether eosinophils could influence the outcome of infection, we monitored pulmonary bacterial loads and survival in B6 $\Delta \mathrm{dblGata}$ mice after Mtb infection. While lung bacterial loads were similar compared to WT mice for the first month, two months after Mtb infection we consistently measured a significant $0.5-1 \log$ increase in the lungs of B6 $\Delta \mathrm{dblGata}$ mice (Fig. 5 A). Moreover, $\triangle$ dblGata mice on either B6 or Balb/C genetic backgrounds succumbed significantly earlier to Mtb infection (Fig. 5 B). To confirm these results in a second, independent genetic model of eosinophil deficiency, we infected transgenic B6 PHIL mice that express cytocidal diphtheria toxin A under the eosinophil-specific EPX promoter (Lee et al., 2004). Like B6 $\Delta$ dbIGata mice, B6 PHIL mice succumbed significantly earlier than WT mice to Mtb infection (Fig. 5 C). When infected with a high dose of $M t b$, both B6 $\Delta$ dblGata (Fig. 5 D) and PHIL 
(Fig. S3 N) mice displayed significantly increased lung bacterial loads at two-three months and succumbed to Mtb infection-induced disease in an infectious dose-dependent manner (Fig. 5 EF). Thus, the data from eosinophil-deficient mouse lines show that eosinophils play a functional role in optimal host resistance during Mtb infection.

To conclude, we found that eosinophils are recruited to Mtb lesions in humans, macaques, zebrafish and mice. In human lung lesions eosinophils were enriched compared to blood and found in TB granulomas. In NHP granulomas eosinophils were present and functionally activated to degranulate. We observed diffuse granule protein staining in the necrotic cores of both human and NHP granulomas, indicative of eosinophil degranulation. In settings associated with tissue necrosis Mtb can survive and propagate extracellularly (Basaraba and Hunter, 2017; Ernst, 2012) perhaps affording eosinophils the opportunity to access and kill extracellular Mtb via their characteristic degranulation and DNA trap formation. Moreover, purified human EPX has been reported to induce Mtb lysis in vitro (Borelli et al., 2003). Alternatively, granulocyte degranulation could facilitate necrotic core formation in granulomas. Thus, the described associations of eosinophils with TB granulomas in human and macaques do not allow for conclusions regarding protective or detrimental roles. In fact, it is likely that the biological function of eosinophils in host resistance to Mtb may be influenced by the stage and dose of infection, disease status and granulomatous features, all factors that vary considerably between the broad host species examined in this study. Nevertheless, we establish here that eosinophils represent an unanticipated integral part of the lung granulocytic response to Mtb across species.

Importantly, our translational approach in mice uncovered an unexpected, yet functional role of eosinophils during murine Mtb infection. Using multiple eosinophil-deficient mouse lines, we show that eosinophils are required for optimal host survival and bacterial control. While the underlying protective mechanisms in mice are currently unclear, they could involve antibactericidal or immunoregulatory effector functions that directly or indirectly affect Mtb growth. However, we show here that neither phagocytosis nor direct infection with Mtb seem likely to be 
occurring at significant levels in eosinophils. Additional non-direct protective mechanisms are therefore likely and could involve immunomodulatory cell-cell interactions between eosinophils and Mtb-infected macrophages or other pulmonary immune cells, maintenance of lung barrier function as well as disease tolerance. Such cell-cell interactions could involve production of lipid mediators, as identified in our gene set enrichment analysis, and type II cytokines. In fact, a recent study in zebrafish showed that non-canonical type II immune IL-4 and IL-13 signals played critical roles in mycobacterial granuloma formation and macrophage epithelialization (Cronan et al., 2021). Additionally, our transcriptional profiling revealed changes in neuronal associated pathways raising the intriguing possibility that eosinophils may also interact with non-immune lung cells such as PNEC and airway sensory neurons. We therefore propose that eosinophil-mediated protection against $M t b$ likely includes multiple non-mutually exclusive mechanisms, that remain to be further experimentally explored in mice and validated in interventional studies in NHPs.

Co-infection with parasitic helminths is common in areas with high TB incidence, and clinical studies have produced contradictory results on the effect of parasitic worm infections on TB outcomes (Babu and Nutman, 2016; Rafi et al., 2012). In some instances of improved clinical outcomes with TB-helminth co-infections (Abate et al., 2015; O'Shea et al., 2018; van Soelen et al., 2012) eosinophils may have contributed to improved outcomes. That said, increased susceptibility to bacterial infections has not been reported in clinical trials of eosinophil-depleting antibodies (Pavord et al., 2012; Rothenberg et al., 2008; Straumann et al., 2010) though longerterm data with examination of patients exposed to Mtb are needed and it is possible that a protective effect of eosinophils is masked in highly heterogenous patient populations.

Taken together, our study represents a multi-species investigation of the eosinophil response to Mtb. Our data reveal that eosinophilic granulocytes, typically associated with type II inflammatory responses, represent an integral part of the granulocyte response to tuberculosis, a disease associated with type I immunity. Thus, our findings open up multiple new lines of 
investigation into the functional relevance of eosinophils during chronic bacterial lung infection and potential new targets for host-directed therapies for tuberculosis.

\section{ACKNOWLEDGEMENTS}

The authors thank the clinical study participants and medical staff at the Khayelitsha Site B CHC in Cape Town, the SPHCC affiliated with Fudan University in Shanghai and the $\mathrm{HCH}$ in Zhengzhou. We thank Drs. Y. Belkaid, A. O'Garra, S. P. Babu, M. Lionakis and A. Sher for data discussions and feedback on the manuscript. We are grateful to Ray Y. Chen for assistance with clinical protocol, all TBIP members, and the staff of the NIAID ABSL2 and ABSL3 facilities. This work was supported in part by the intramural research program of NIAID (K.D.M-B, D.L.B, C.E.B.3rd, L.E.V.) and the National Natural Science Foundation of China grant (\#81770010 to KW.W.) R.J.W is supported by NIH (U01Al115940), Wellcome $(104803,203135)$ and FC0010218 (CRUK, UKRI and Wellcome).

\section{Declaration of Interests:}

The authors declare no competing interests.

FIGURE LEGENDS

Figure 1: Eosinophils are decreased in circulation and enriched in human lung lesions during tuberculosis.

(A) Cape Town cohort: Circulating eosinophil and neutrophil numbers in healthy control (HC, $\mathrm{n}=20$ ), interferon gamma release assay (IGRA) positive latently Mtb-infected individuals (LTBI, $\mathrm{n}=77$ ) and IGRA positive and GeneXpert (GXPT) positive pulmonary TB (PTB, $\mathrm{n=48}$ ) individuals (Kruskal-Wallis with Dunn's correction) (B) Cape Town Cohort: Circulating eosinophil numbers at baseline $(\mathrm{BL})$ and after anti-tubercular treatment (ATT) (Wilcoxon-matched pairs test, two tailed) 
(n=37) (C) Shanghai Cohort: Representative FACS plots (from ID\#1) of granulocytes in whole blood (WB) and resected human TB lesions ( $n=9$, color coded) (D) Shanghai Cohort: Summary eosinophil and neutrophil ratios $(E / N)$ in resected human lung lesions (connecting line according to color coded patient ID, tissues are depicted as mean and SEM of $n=1-6$ samples per tissue type and patient) (E) Shanghai Cohort: Eosinophil (circles) and neutrophil (triangles) proportions of $\mathrm{CD}_{4} 5^{+}$cells depicting individual samples per patient (connecting line, patient IDs are color coded, Wilcoxon-matched pairs test, two tailed) (F) Shanghai Cohort: eosinophil and neutrophil ratios $(\mathrm{E} / \mathrm{N})$ in ${ }^{18} \mathrm{FDG} \mathrm{PET} / \mathrm{CT}$ low or high signal $\left(\mathrm{SUV}_{\max }>5.0\right)$ intensity lung lesions (connecting line, $n=5$, patient IDs color coded, Ratio paired t-test, two-tailed) (G) Shanghai Cohort: Summary eosinophil and neutrophil ratios $(E / N)$ in combined resected human lung lesions $(n=3=22)$ per patient $(n=6)$ compared to patients' blood eosinophils (connecting line, patient IDs color coded, Ratio paired t-test, two-tailed)

Figure 2: The presence of eosinophils in mycobacterial granulomas is evolutionarily conserved.

(A) Rome cohort: Hematoxylin and Eosin (H\&E) and eosinophil peroxidase (EPX) immunostaining of paraffin embedded human Mtb lung lesions; arrows indicate eosinophils. (B) H\&E and EPX immunostaining of paraffin embedded rhesus macaque Mtb granulomas, arrows indicate eosinophils. (C) Organized core of a multifocal granuloma in the ovary of an $M$. marinum infected zebrafish. Black arrowheads indicate individual eosinophils stained by Periodic-acidSchiff (PAS) in the rim of the granuloma.

Figure 3: Eosinophils infiltrate and degranulate in Mtb granulomas of rhesus macaques.

(A) Representative FACS plots of EPX staining of eosinophils in whole blood (WB) from healthy donors and uninfected rhesus macaques WB and bronchoalveolar lavage (BAL) (B) Animal ID list and corresponding color code of Mtb infections in present study (two independent studies, 
$\mathrm{n}=3-4, M+F)$ and percent eosinophils in pulmonary Mtb granulomas $(n=46)(C)$ Correlation plot of eosinophil frequency and granuloma Mtb CFU (Spearman) (D) Left: Representative FACS plots of eosinophil CD63 surface expression to quantify degranulation in indicated tissues, Right: Summary data on frequency of degranulated eosinophils per individual granulomas $(n=46)$ (Wilcoxon-Matched Pairs test for WB and BAL comparison, granuloma comparisons Ratio matched pared t-test, two tailed). (E) Correlation plot of frequency of $\mathrm{CD}_{63}{ }^{+}$eosinophils and eosinophil abundance (Spearman). (F) Correlation plot of frequency of CD63+ eosinophils and granuloma Mtb CFU (Spearman).

Figure 4: Pulmonary transcriptional profiling of Mtb-infected eosinophil-deficient mice reveals perturbations in lung neuronal associated pathways.

(A) Cell number of lung parenchymal (CD45 i.v. ${ }^{\text {neg }}$ ) eosinophils over time after standard low dose (100-300 colony forming units (CFU) aerosol Mtb H37Rv infection in B6 mice $(M+F, n=12-25$ per time-point, 3-4 independent experiments, Mann-Whitney) (B) Representative FACS plots of intravascular (i.v.) staining and Mtb-mCherry quantification of eosinophils in the lungs of B6 WT mice after Mtb aerosol infection at d30 (3-4 independent experiments) (C) Representative sections of H\&E staining from paraffin-embedded lungs of Mtb-infected (d90) WT or B6 $\Delta \mathrm{dblGata}$ mice (M+F, n=5-6, two independent experiments) (D) Heatmap of top 20 up- and top 20 downregulated DEGs of d90 WT compared to d90 B6 $\Delta \mathrm{dblGata}$ normalized to uninfected WT mice $(F$, $\mathrm{n}=4-5$, one experiment). Gene annotation as follows: Neuronal associated genes (pink), lipid and short chain fatty acid metabolism (green) (E) Gene set enrichment analysis based on Reactome (top) and KEGG (Bottom) of key pathways that are selectively downregulated in d90 B6 $\Delta \mathrm{dblGata}$ mice. Neuronal associated pathways are highlighted in pink, lipid and short chain fatty acid metabolism pathways are highlighted in green. 
Figure 5: Eosinophil deficiency in mice results in increased susceptibility to Mtb infection.

(A) Colony forming units (CFU) over time in lungs of standard low dose Mtb H37Rv (100-300 CFU) infected WT B6 or B6 $\Delta$ dbIGata mice $(M+F, n=11-21,2-3$ experiments per timepoint; MannWhitney) (B) Survival of WT Balb/C or B6 or $\Delta$ dblGata with LD Mtb (100-300 CFU) infection (M+F, LD n=5-12, 2-3 experiments each; Mantel-Cox) (C) Survival of WT B6 or PHIL mice after infection with 100-300 CFU Mtb (M+F, $\mathrm{n}=4-8,2$ experiments each; Mantel-Cox) (D) Lung CFU 80 days after high dose (1000-1500 CFU) Mtb infection of WT B6 or B6 $\Delta$ dblGata mice (M+F, n=1121, 3 experiments; Mann-Whitney) (E) Survival of WT Balb/C or B6 or $\Delta$ dblGata with 1000-1500 CFU Mtb infection ( $\mathrm{n}=13-27,3-4$ experiments each; Mantel-Cox).(F) Survival of WT B6 or PHIL mice after infection with 1000-1500 CFU Mtb (M+F, n=17-18, 3 experiments; Mantel-Cox).

\section{Supplemental Figure S1: Peripheral blood eosinophils in clinical cohorts and granulocyte isolation of from human TB lung lesions.}

(A) Cape Town Cohort: Circulating neutrophil numbers and (B) eosinophil/neutrophil (E/N) ratio in interferon gamma release assay (IGRA) negative healthy controls ( $H C, n=20$ ), IGRA positive latently Mtb-infected individuals $(\mathrm{LTBI}, \mathrm{n}=77$ ) and GeneXpert (GXPT) positive pulmonary TB (PTB, $\mathrm{n}=49$ ) individuals (Kruskal-Wallis with Dunn's correction) (C) Zhengzhou Cohort: eosinophil/neutrophil $(E / N)$ ratio in $\mathrm{HC}(n=30)$, acid fast bacilli (AFB) staining neg (AFB-, sputum negative), PTB ( $n=64$, pink), and AFB positive (AFB+, sputum positive) PTB ( $n=48$, blue), and extrapulmonary TB (EPTB, $n=50$, yellow) (Kruskal-Wallis with Dunn's correction) (D) Zhengzhou Cohort: Circulating eosinophil numbers at baseline $(\mathrm{BL})$ and 2 wks. after anti-tubercular treatment (ATT) (Matched Wilcoxon) from color coded clinical groups (pink, IGRA+AFB-PTB, n=47 pairs; blue, IGRA+AFB+PTB, n=34 pairs; yellow, EPTB, $n=30$ pairs). (E) London Cohort: Circulating eosinophil numbers in survivors $(n=901)$ or deceased $(n=55)$ TB patients (Mann-Whitney). (F) London Cohort: Circulating eosinophil numbers correlated with time to death (Spearman correlation, $n=49$ ). (G) Schematic study overview and examples of human lung TB lesions after 
resection surgery $(n=9)$ and flow cytometric gating strategy of human lung TB lesions for eosinophil and neutrophil quantification in Shanghai cohort. (H) Shanghai Cohort: Eosinophil (circles) and neutrophil (triangles) proportions of $\mathrm{CD} 45^{+}$cells depicting individual samples per patient (connecting line, patient IDs are color coded, Wilcoxon-matched pairs test, two tailed) (I) Shanghai Cohort: Summary eosinophil and neutrophil ratios $(E / N)$ in resected human lung lesions (connecting line according to color coded patient ID, tissues are depicted as mean and SEM of $\mathrm{n}=1-6$ samples per tissue type and patient). (J) Shanghai $(\mathrm{SH})$ Cohort: Eosinophil (circles) and neutrophil (triangles) proportions of $\mathrm{CD} 45^{+}$cells in ${ }^{18} \mathrm{FDG}$ PET/CT low or high signal $\left(\mathrm{SUV}_{\max }>\right.$ 5.0) intensity lung lesions (connecting line, $n=5$, patient IDs color coded, Wilcoxon-matched pairs test, two tailed).

\section{Supplemental Figure S2: Human and NHP TB lesions assessed by H\&E and EPX immunohistological and flow cytometric staining.}

(A) Rome cohort: Histological scoring of eosinophil distribution in human TB lesion on blinded specimens $(n=10)$. (B) Animal IDs and infection dose and strains for NHP Mtb infections $(n=7$, $M+F)$, two independent experiments (C) Granuloma outline and legend for histological scoring of NHP granulomas on blinded specimens ( $n=5)$. (D) EPX immunofluorescence staining on thin sections from paraffin embedded granulomas after Erdman-mCherry (blue) Mtb infection. (E) Isotype and fluorescence minus one FMO (empty) control of EPX-AF488 staining in NHP whole blood (WB). (F) Example EPX FACS staining in granulomas from rhesus macaques.

\section{Supplemental Figure S3: Transcriptional changes and immune profiling in eosinophil deficient mice after Mtb infection.}

(A) Example flow cytometry of eosinophils in B6 WT and $\Delta$ dbIGata mice and lung eosinophil frequency in naïve and Mtb infected (100-300 CFU) B6 WT and eosinophil deficient mice. (M+F, $\mathrm{n}=3-28,1-4$ independent experiments per timepoint, Mann-Whitney) (B) Numbers of total and 
lung parenchymal (i.v. neg) eosinophils at indicated time points after $M$ tb infection $(M+F, n=12-$ 25, 3-4 independent experiments per timepoint, Mann-Whitney) (C) Representative flow cytometry for intravascular stain and ESAT6 ${ }_{4-17}$ CD4 tetramer with quantification of Mtb-specific CD4 $^{+} \mathrm{T}$ cells and (D) Mtb-specific CD8+ T cells 4 weeks p.i. $(M+F, n=8-10$, Mann-Whitney, 2 experiments). Quantification of (E) total CD4+ cells and ESAT6 4 -17 CD4 tetramer+ CD4+ T cells and $(\mathbf{F})$ total CD8+ and $10.4_{4-11}$ specific CD8 tetramer+ cells three months after infection $(\mathrm{M}+\mathrm{F}$, $\mathrm{n}=5-6$, Mann-Whitney, 2 experiments). Quantification at three months post infection of $(\mathbf{G}) \mathrm{Nk} 1.1^{+}$ cells, (H) neutrophils, (I) interstitial macrophages (Macs)/dendritic cell 2 (DC2) and their corresponding frequency of arginase (Arg1) or iNOS (Nos2) expression (M+F, n=5-6, MannWhitney, 2 experiments). Quantification at baseline and three months post infection of $(\mathbf{J})$ alveolar macrophages $(\mathrm{AM})$ and $(\mathrm{K}) \mathrm{XCR} 1+$ conventional dendritic cells 1 (cDC1) ( $\mathrm{F}, \mathrm{n}=4-6$, MannWhitney, 1 experiment). (L) Volcano plot of differentially expressed genes (DEG, red) between lungs of d90 WT $(n=4)$ or B6 $\Delta$ dbIGata $(n=5)$ Mtb-infected mice. Green are genes with Log2 Fold Change \pm 1.4 , Blue are genes with a $p$-value and False discovery rate (FDR) lower than 0.05 and grey genes are not significant. The changes in gene expression levels were considered significant when statistical test values (FDR adjusted p-value) were lower than 0.05 and the fold change/difference higher than \pm 1.4 . Neuronal associated genes are annotated in pink. One experiment with $n=4=5$ per group. (M) Venn diagram of DEG in lungs from three-month (d90) infected WT B6 $(n=4)$ or B6 $\Delta$ dblGata mice $(n=5)$ and uninfected (d0) B6 $\Delta$ dblGata $(n=4)$ compared to lungs from uninfected (d0) WT B6 mice $(n=5)$. Upregulated DEG are shown in red and downregulated DEG in blue. One experiment with $n=4=5$ per group (N) Lung bacterial loads 85 days after high dose Mtb infection in eosinophil deficient B6 PHIL mice (M, n=4-5, MannWhitney, 1 experiment). 


\section{METHODS}

\section{Clinical study population in Shanghai, CN}

Human lung resection material was obtained at the Shanghai Public Health Clinical Center (SPHCC) between 2015 and 2019 following approval by the SPHCC Ethics Committee (\# 2015S046-02 and 2019-S009-02). All patients provided written informed consent. Clinically indicated lung or pleural tissue resection surgery was performed at SPHCC as an adjunctive therapy for TB (L.W, Y.S.). Patients with HIV, other ongoing infections or immunodeficiency conditions were excluded. Using sterile instruments, resected lung tissue samples from participants ID\#1-4 were divided based on macroscopically apparent diverse tissue pathologies, and a minimum of triplicate samples for each pathologic subtype were used for cell isolation and flow cytometric analysis. Participants ID\#5-9 consented to ${ }^{18} \mathrm{FDG}$ PET/CT signal scanning 1-6 days prior to clinically indicated surgery to guide resection and minimize risk of postoperative complications, such as increasing bronchial stump and lung resection margin embedding. After resection lung samples were further dissected based on standardized uptake values (SUV) into maximum SUV (PET high, SUV $\max >5.0$ ) and low (PET low, similar to background SUV) signal pieces according to mapped 18FDG PET/CT scanning results using sterile scissors. Triplicate samples were generated for cell isolation and flow cytometric analysis for PET high and low regions from the same individuals. FACS studies were performed onsite by K.D.M-B, Z.H. and K-W.W. and samples were acquired on an LSRFortessa (BD Biosciences) and analyzed with FlowJo 10 (BD Biosciences, FlowJo, LLC).

ID\#1: 28 yr. old non-smoker male, sputum smear and culture negative, received repeated ATT for 8 years, drug resistance was not detected. Treatment before surgery: isoniazid, rifampicin, pyrazinamide, and ethambutol. Surgical indication: presence of cavities and irreversible TB progression with lung destruction. Postoperative pathologic diagnosis: TB with large cavity with aspergilloma, extensive fibrotic lesions with a mixture of local cavities, 
granulomas, consolidations, and caseous necrosis. Acid-fast staining was 4+ positive and culture was positive for Mtb.

ID\#2: 22 yr. old non-smoker male, sputum smear and culture negative, drug resistance was not detected. Treatment before surgery: four-month treatment with isoniazid, rifampicin, pyrazinamide, ethambutol, and levofloxacin. Surgical indication: enlargement of pleural empyema. Postoperative pathologic diagnosis: presence of inflammatory cells, fibrosis, coagulative necrosis, caseous necrosis. Acid-fast smear staining was 2+ positive and culture was positive for Mtb.

ID\#3: 25 yr. old non-smoker female; sputum smear and culture negative at time of surgery; sputum and culture confirmed diagnosis of pulmonary tuberculosis in both lungs in 2010, repeated ATT over 6 years; drug resistance to streptomycin, isoniazid, levofloxacin and para-aminosalicylic acid were detected. Despite isoniazid, rifampicin, pyrazinamide and ethambutol regimen treatment for 1 year re-examination of chest CT showed bilateral lesions were found to be progressing and sputum culture was positive. Treatment before surgery: cycloserine, amikacin, linezolid, clofazimine, moxifloxacin, isoniazid, rifampicin, pyrazinamide, and ethambutol. Surgical indication: the presence of multi-drug resistance and cavities in the lower right lobe of the lung with complication of pneumothorax. Postoperative pathologic diagnosis: tissue infiltration of inflammatory cells, epithelioid granuloma formation, caseous necrosis and acid-fast staining (4+), which was not culture positive.

ID\#4: 32 yr. old non-smoker female, 5 years of ATT with extended drug resistance to streptomycin, isoniazid, rifampicin, and ofloxacin; sputum was 4+ smear-positive and culture positive. Surgical indication: presence of extended drug resistance and consolidations and atelectasis in the lower right lobe of the lung. Postoperative pathologic diagnosis: inflammatory cell infiltration, epithelioid granuloma formation, caseous necrosis and acid-fast staining (2+), which was culture positive for Mtb. 
ID\#5: 20 yr. old non-smoker female, drug resistance was not detected, positive sputum smear, anti-TB antibody and T-spot. Sputum smear positive in July 2018 and treatment before surgery: 7-month ATT with isoniazid, rifampicin, pyrazinamide and ethambutol. Surgical indication: presence of tuberculous pleurisy related tumor with increasing size over preceding 3 months Postoperative pathologic diagnosis: inflammatory cell infiltration, epithelioid granuloma formation and coagulation necrosis. Acid-fast smear staining (4+)

ID\#6: 38 yr. old male smoker (15 yrs. history), sputum smear and culture negative at time of surgery, no drug resistance, nine years prior to surgery received a one-year ATT with isoniazid, rifampicin, pyrazinamide and ethambutol after acid-fast smear positive with CT showing improved TB lesions after treatment. Symptoms relapsed three years prior to surgery, and he received ATT with isoniazid, rifampicin, pyrazinamide, ethambutol, levofloxacin, azithromycin. Surgical indication: presence of repeated TB-related symptoms including tuberculous pleuritis and cavities. Postoperative pathologic diagnosis: epithelioid granuloma and coagulative necrosis. Tissue sample acid-fast smear staining was negative.

ID\#7: 39 yr. old male smoker (20 yr. history) with 5 yr. history of TB; no drug resistance, sputum smear negative at time of surgery. Previously treated with isoniazid, rifampicin, pyrazinamide and ethambutol regimen with positive sputum smear in local hospital in August 2014 for 1 year, tuberculosis recurred in June 2016, and re-anti-tuberculosis treatment for 1 year; in April 2018, the patient developed hemoptysis and chest CT suggested left upper lobe tuberculosis with cavities. He received a third anti-tuberculosis treatment. Treatment before surgery: 15-month ATT with isoniazid, rifampicin, pyrazinamide and ethambutol TB-Ab-IgG was positive. Surgical indication: clinical diagnosis of tuberculous and persistent TB cavity in spite of ATT. Postoperative pathologic diagnosis: tissue infiltration of inflammatory cells, granuloma formation, coagulative necrosis, calcification, focal pulmonary hemorrhage and local fungal infection. Acid-fast smear staining was negative and Periodic-acid-Schiff (PAS) staining was positive. 
ID\#8: 57 yr. old non-smoker male, sputum smear and anti-TB antibody were positive, rifampicin resistance was detected by using GeneXpert (Cepheid, Sunnyvale, CA). Treatment before surgery: 1-month ATT with rifampicin, pyrazinamide, ethambutol, isoniazid and levofloxacin (stopped due to cutaneous pruritus) and 1-month treatment with rifapentine. Surgical indication: presence of lung cavity. Postoperative pathologic diagnosis: inflammatory cell infiltration, epithelioid granuloma formation and coagulation necrosis. Fluid from postoperative lesion was acid-fast smear 3+, and GXPT-positive for Mtb and rifampicin sensitive.

ID\#9: 23 yr. old non-smoker female, diagnosed with pulmonary tuberculosis in 2016 (positive sputum smear), isoniazid, rifampicin, pyrazinamide and ethambutol anti-tuberculosis treatment was discontinued after 6 months, fatigue, night sweats, and large pleural effusion occurred in 2018, and the sputum smear was positive again and no drug resistance prior ATT. Treatment before surgery: 10-month ATT with isoniazid, rifampicin, ethambutol, levofloxacin, bicyclol and amikacin. Surgical indication: clinical diagnosis of tuberculous hydrothorax and destructive pneumophthisis. Postoperative pathologic diagnosis: tissue infiltration of inflammatory cells, epithelioid granuloma formation, fibrosis and coagulative necrosis. GXPT test on intraoperative endocrine resected tissue revealed rifampicin sensitive $M t b$ complex.

\section{Clinical Study populations in Cape Town, ZA}

For eosinophil studies from Cape Town, ZA, participants were recruited from the Site B Community Health Centre (CHC), in Khayelitsha between March 2017 and December 2018. Some study participants are described elsewhere (Du Bruyn et al., 2020) and in the table below. Briefly, all participants were HIV-uninfected adults (age $\geq 18 \mathrm{yr}$.) and who provided written informed consent. The study was approved by the University of Cape Town Human Research Ethics Committee (HREC 050/2015) and was conducted under DMID protocol no.15-0047. Those in the active TB group (PTB, $n=48$ ) all tested sputum GeneXpert Mtb/RIF (GeneXpert (GXPT), Cepheid, Sunnyvale, CA) and/or sputum Mtb liquid culture positive. All active TB cases were drug 
sensitive and had received no more than one dose of ATT at the time of baseline blood sampling.

The latent TB group (LTBI, $\mathrm{n}=77$ ) were all asymptomatic with a positive IFNy release assay (IGRA, QuantiFERON®-TB Gold In-Tube), tested sputum GXPT Mtb/RIF negative and had no clinical or radiographic evidence of active TB. Similarly, the healthy control group $(\mathrm{HC}, \mathrm{n}=20)$ were all asymptomatic, without history of previous TB, tested sputum GXPT Mtb/RIF negative, had no clinical or radiographic evidence of active TB and tested IGRA negative. Sputum GXPT Mtb/RIF and differential full blood count were performed by the South African National Health Laboratory Services.

\begin{tabular}{|c|c|c|c|}
\hline Cape Town & $\begin{array}{l}\text { Healthy Control (HC); } \\
\text { IGRA - }\end{array}$ & $\begin{array}{l}\text { Latent TB Infection } \\
\text { (LTBI); IGRA+ }\end{array}$ & $\begin{array}{l}\text { Active Pulmonary TB } \\
\text { (PTB); Sputum GXPT + }\end{array}$ \\
\hline N (HIV negative) & 20 & 77 & 48 \\
\hline Average age (years) & 38 & 33 & 37 \\
\hline Gender (F/M) & $12 / 8$ & $39 / 38$ & $13 / 35$ \\
\hline
\end{tabular}

\section{Clinical Study population in Zhengzhou, CN}

HIV uninfected individuals with symptoms indicative of active TB who were administered less than two weeks of ATT were enrolled into a natural history study to assess response to chemotherapy (NCT01071603) conducted at the Henan Chest Hospital (HCH) in Zhengzhou from 2010 to 2012. For detailed descriptions of the clinical cohort see Mayer-Barber et al. 2014 and others (Trauner et al., 2017; Vinhaes et al., 2019).

\section{Clinical Study population in London, UK}

The clinical study population of the TB patients in London, who were retrospectively identified by database and case-note review at the King's College Hospital and Newham University Hospital Trust, has been described in detail in Lowe et al. (Lowe et al., 2013). 


\section{Clinical autopsy cohort from Rome, Italy}

The autopsy-cohort from the National Institute for Infectious Diseases, "Lazzaro Spallanzani" (INMI) in Rome, Italy included neutral buffered-formalin fixed, paraffin-embedded autopsy lung tissue samples from patients with histologically, histochemically, or PCR-proven confirmed pulmonary TB as previously described in Blauenfeldt et al. (Blauenfeldt et al., 2018).

\section{Clinical Study population in Bethesda, USA}

For whole blood flow cytometry or in vitro experiments on eosinophils, healthy controls from the NIH Clinical Center (Bethesda, MD) were recruited on an institutional review board-approved clinical protocol to obtain normal blood samples (NCT00001846). All participants gave written informed consent.

\section{Rhesus Macaques}

Thirteen healthy $>2$-year-old rhesus macaques (male and female, tuberculin skin test negative) were received from the NIAID breeding colony on Morgan Island. Animals were housed in nonhuman primate biocontainment racks and maintained in accordance with the Animal Welfare Act, the Guide for the Care and Use of Laboratory Animals and all applicable regulations, standards, and policies in a fully AAALAC International accredited Animal Biosafety Level 3 vivarium. All procedures were performed utilizing appropriate anesthetics as listed in the NIAID DIR Animal Care and Use Committee approved animal study proposal LPD25E. Euthanasia methods were consistent with the AVMA Guidelines on Euthanasia and endpoint criteria listed in the NIAID DIR Animal Care and Use Committee approved animal study proposal. 


\section{Mtb infections of rhesus macaques}

Rhesus macaques from two independent infections provided tissues for this study (Fig. S4 B) and animals DF4A, 0DH, DF8B, DFNO have previously been reported in another study (Sallin et al., 2018). Individual macaques were infected with 30-50 colony forming units (CFU) of either H37Rv or mCherry-expressing Erdman strain of Mtb. For infections, animals were anesthetized and $2 \mathrm{ml}$ of $M t b$ containing saline were instilled bronchoscopically into the right lower lung lobe. Delivered infection doses were confirmed by plating aliquots of instillation solution onto $7 \mathrm{H} 11$ agar plates. Colony counts were determined after 21 days.

\section{Mice}

C57BL/6 (B6) mice were purchased from Taconic Farms (Hudson, NY). B6 PHIL mice (Lee et al., 2004) were obtained from Elizabeth Jacobsen (Mayo Clinic, Pheonix, AZ). B6 $\triangle$ dbIGata (JAX 33551, B6.129S1(C)-Gata1tm6Sho/LvtzJ) mice were provided by Helene Rosenberg (NIAID). $\triangle$ dbIGata (JAX 5653, C.129S1(B6)-Gata1tm6Sho/J) on the BALB/CJ background mice were purchased from Jackson Laboratories (Bar Harbor, ME). Both male and female mice, 8-24 wk. old at the onset of experiments, were used, and experimental groups in individual experiments were age and sex matched. All animals were bred and maintained in an AAALAC-accredited ABSL2 or ABSL3 facility at the $\mathrm{NIH}$, and experiments were performed in compliance with an animal study proposal approved by the NIAID Animal Care and Use Committee (protocol LCIM17E).

\section{Mtb infections of mice}

For aerosol infection of mice with H37Rv strains of Mtb, animals were placed in a whole-body inhalation exposure system (Glas-Col, Terre Haute, IN) and exposed to aerosolized Mtb. Delivery doses (100-300 CFU for standard low dose, 1000-1500 CFU for high dose) were confirmed by measuring lung bacterial loads at 2-24 hrs post-exposure in control mice. Bacterial loads were 
measured in tissue homogenates obtained via digestion and dissociation using GentleMacs (Miltenyi Biotec, CA) or mechanical homogenization using Precellys Evolution (Precellys, Atkinson, NH). Lung homogenates were serially diluted in PBS/Tween-20 and cultured on Middlebrook 7H11 agar plates supplemented with oleic acid-albumin-dextrose-catalase (Difco, Detroit, MI). Colony counts were determined after 21 days. H37Rv-mCherry was provided by Kevin Urdahl (Cohen et al., 2018).

\section{M. marinum infection and paraffin embedding of zebrafish}

All zebrafish husbandry and experiments were approved by the Duke University Animal Care and Use Committee (protocol A122-17-05). Single cell aliquots of cerulean-expressing M. marinum were made according to the method of Takaki (Takaki et al., 2013). Zebrafish were anesthetized with $0.016 \%$ tricaine and infected with $M$. marinum-expressing cerulean fluorescent protein at a dose of 400 fluorescent bacteria/animal by intraperitoneal injection of the zebrafish. Zebrafish were maintained in spawning tanks with daily water changes. At 2 weeks post infection, zebrafish were euthanized by tricaine overdose and the head and the tail of the zebrafish were removed with a scalpel. A small incision was made in the belly of the zebrafish and the zebrafish was fixed for 2 days in $4 \%$ paraformaldehyde. The animals were subsequently processed and paraffinembedded by the Duke Research Immunohistology Laboratory.

\section{Histopathology}

Tissue samples were fixed with $4 \%$ paraformaldehyde, paraffin-embedded, sectioned and stained with either hematoxylin and eosin (H\&E), the Ziehl-Nielsen histochemical method to visualize acid-fast mycobacteria, anti-EPX for the immunohistochemical identification of eosinophils or Periodic-acid-Schiff (PAS) for necrosis. Eosinophils were identified via light microscopy using an Olympus BX51 microscope (magnifications of 4x, 10x or 40x) and photomicrographs were taken using an Olympus DP73 camera. The distribution of eosinophil staining was assessed 
microscopically using indicated scales; all histological analyses were scored in a blinded manner by a board-certified veterinary pathologist.

\section{Confocal microscopy of rhesus macaque granulomas}

Excised granulomas from Erdman-mCherry fluorescent Mtb-infected rhesus macaques were fixed overnight in 4\% paraformaldehyde and subsequently embedded in Optimal cutting temperature compound (OCT, Fisher Scientific) and stored at $-80^{\circ} \mathrm{C}$. Cryostat cut sections $(7 \mu \mathrm{m})$ were placed onto microscope slides (Fisher Scientific) and sections were blocked with 10\% FCS in PBS and incubated with anti-Laminin-AF647 and anti-human EPX AF488 for $1 \mathrm{~h}$ incubation at $37^{\circ} \mathrm{C}$ in a humidified chamber. Afterwards the slides were washed in PBS for 10 min and mounted with ProLong® Gold Antifade mountant (ThermoFisher). The samples were analyzed with a Leica SP8 confocal laser fluorescence microscope and Imaris software (Bitplane).

\section{RNA extraction and RNA-Seq}

For RNA extraction dedicated lung lobes were placed in RNAlater (Invitrogen, Carlsbad, CA) and stored at $-80^{\circ} \mathrm{C}$. RNAlater-stabilized lung lobes were thawed at RT for $20 \mathrm{~min}$, then homogenized in RLT plus buffer with $\beta$-mercaptoethanol (Qiagen, Hilden, Germany). Total RNA was then isolated from the RLT-homogenized cells using the RNeasy Plus Mini Kit (Qiagen, Hilden, Germany). RNA quantification, qualification as well as library preparation and transcriptome sequencing was performed by Novogene (Novogene Corporation Inc, Sacramento, CA). Briefly, RNA degradation and contamination was monitored on $1 \%$ agarose gels and RNA purity was checked using the NanoPhotometer ${ }^{\circledR}$ spectrophotometer (IMPLEN, CA, USA). RNA integrity and quantitation were assessed using the RNA Nano 6000 Assay Kit of the Bioanalyzer 2100 system (Agilent Technologies, CA, USA). A total amount of $1 \mu \mathrm{g}$ RNA per sample was used as input material for the RNA sample preparations. Sequencing libraries were generated using NEBNext® 
UltraTM RNA Library Prep Kit for Illumina ${ }^{\circledR}$ (NEB, USA) following manufacturer's recommendations and index codes were added to attribute sequences to each sample. Briefly, mRNA was purified from total RNA using poly-T oligo-attached magnetic beads. Fragmentation was carried out using divalent cations under elevated temperature in NEBNext First Strand Synthesis Reaction Buffer (5X). First strand cDNA was synthesized using random hexamer primer and M-MuLV Reverse Transcriptase (RNase H-). Second strand cDNA synthesis was subsequently performed using DNA Polymerase I and RNase $\mathrm{H}$. Remaining overhangs were converted into blunt ends via exonuclease/polymerase activities. After adenylation of 3' ends of DNA fragments, NEBNext Adaptor with hairpin loop structure were ligated to prepare for hybridization. In order to select cDNA fragments of preferentially 150 200 bp in length, the library fragments were purified with AMPure XP system (Beckman Coulter, Beverly, USA). Then $3 \mu$ l USER Enzyme (NEB, USA) was used with size-selected, adaptor ligated cDNA at $37^{\circ} \mathrm{C}$ for 15 min followed by 5 min at $95^{\circ} \mathrm{C}$ before PCR. Then PCR was performed with Phusion High-Fidelity DNA polymerase, Universal PCR primers and Index (X) Primer. At last, PCR products were purified (AMPure XP system) and library quality was assessed on the Agilent Bioanalyzer 2100 system. The clustering of the index-coded samples was performed on a cBot Cluster Generation System using PE Cluster Kit cBot-HS (Illumina) according to the manufacturer's instructions. After cluster generation, the library preparations were sequenced on an Illumina Novaseq 6000 platform and paired-end 150bp reads were generated. The entire gene expression data set will be available at the GEO database accession number GSE165871.

\section{Transcriptional analysis}

For all samples, low-quality bases were removed and adapters were trimmed using Trimmomatic V0.32 (Bolger et al., 2014). After the quality check, sequences were aligned to the Mus musculus genome (GRCm38.p6 version 67), with STAR2.7.0 (Dobin et al., 2013). After mapping, the output was converted to count tables with the tximport package (Soneson et al., 2015) from $R$ 4.0.2. The 
stratification was performed as follows: d0 WT B6 group was composed of 5 samples: d0WT1d0WT5, d0 $\Delta$ dbIGata group (d0Gata1-4), d90 WT B6 group (d90WT1-4) and d90 $\Delta$ dbIGata group (d90Gata1-5). Count gene expression matrix was examined by DESeq2 packed (Love et al., 2014) from $R$ 4.0.2 to identify differentially expressed genes (DEG) following the comparisons: d90 $\Delta$ dblGata vs. d90 WT B6, d0 $\Delta$ dblGata vs. d0 WT B6, d90 $\Delta$ dbIGata vs. d0 WT B6, d90 WT B6 vs. d0 WT B6. Changes in gene expression levels were considered significant when statistical test values (FDR adjusted p-value) were lower than 0.05 and the fold change/difference higher than \pm 1.4 . Candidate DEG were visualized in volcano plots and Venn diagrams with EnhancedVolcano and VennDiagram packages from $R$ 4.0.2. The obtained DEG were scanned by REACTOME (Yu and He, 2016) and KEGG (http://www.genome.jp/kegg/) pathway database using compareCluster package (Yu et al., 2012) from $R$ 4.0.2. The gene list of interferon-inducible (Type I) and IFN-y-inducible (Type II) lung modules were retrieved from Singhania et al. (Singhania et al., 2019). The sample clustering and classification were assessed using Heatmaps, applied in the variance stabilizing transformation gene expression values.

\section{Cell Isolations for flow cytometry - Peripheral blood}

Rhesus macaque and human blood samples were collected in EDTA tubes and whole blood was used for flow cytometry. Briefly, antibody-cocktails were directly added to $200 \mu$ of whole blood and stained at $37^{\circ} \mathrm{C}$ for $20 \mathrm{~min}$, after which samples were washed twice in $4 \mathrm{ml} 1 \% \mathrm{FCS} / \mathrm{PBS}$. Fixable live/dead cell stain (Molecular Probes-Invitrogen) was used according to the manufacturer's protocol. Pellets were then fixed and permeabilized with the Foxp3 Transcription Factor Staining Buffer Kit (Life Technologies/eBioscience) for at least $1 \mathrm{hr}$ followed by intracellular antigen staining for $30 \mathrm{~min}$ at $4^{\circ} \mathrm{C}$. Cells were washed and samples acquired on a FACSymphony (BD Biosciences) at NIH or a LSRFortessa (BD Biosciences) at SPHCC. FACS data were analyzed using FlowJo 10 (BD Biosciences, FlowJo, LLC). 


\section{Cell Isolations for flow cytometry - Bronchoalveolar lavage (BAL) and granulomas from} rhesus macaques

Rhesus macaque BAL samples were passed through a $100 \mu \mathrm{m}$ cell strainer, pelleted, and counted for analysis. Granulomas were individually resected from the lungs and samples used for flow cytometry analysis were pushed through a $100 \mu \mathrm{m}$ cell strainer. Aliquots from all samples were serially diluted and plated on 7H11 agar plates for CFU quantification. Alternatively, resected granulomas were fixed in $4 \%$ paraformaldehyde for later paraffin embedding. Samples were stained with surface antibody-cocktails for $20 \mathrm{~min}$ at $4^{\circ} \mathrm{C}$, followed by fixable live/dead cell stain (Molecular Probes-Invitrogen). Cells were then fixed and permeabilized (eBioscience Transcription Factor Staining Buffer Kit) for at least $1 \mathrm{hr}$ followed by intracellular antigen staining for $30 \mathrm{~min}$ at $4^{\circ} \mathrm{C}$. Cells were washed and samples acquired on a FACSymphony (BD Biosciences) at NIH. FACS data were analyzed using FlowJo 10 (BD Biosciences, FlowJo, LLC).

\section{Cell Isolations for flow cytometry - Human and murine lung tissue}

Mice were intravenously (i.v.) injected $3 \mathrm{~min}$. prior to euthanasia with $5 \mu \mathrm{g} / \mathrm{mouse}$ of APC or BV711 labelled CD45 (30-F11) as previously reported (Anderson et al., 2014). Lungs from infected mice were digested using the Miltenyi lung cell isolation buffer and dissociated via GentleMACS (Miltenyi Biotec, CA) according to the manufacturer's instructions. Digested lung was fully dispersed by passage through a $100 \mu \mathrm{m}$ pore size cell strainer and an aliquot was removed for bacterial load measurements. Cells were then washed and purified using a $37 \%$ Percoll step. Samples were stained with surface antibody-cocktails for $20 \mathrm{~min}$ at $4^{\circ} \mathrm{C}$ followed by fixable live/dead cell stain (Molecular Probes-Invitrogen). Cells were then fixed and permeabilized (eBioscience Transcription Factor Staining Buffer Kit) for at least $1 \mathrm{hr}$ followed by intracellular antigen staining for $30 \mathrm{~min}$ at $4^{\circ} \mathrm{C}$. Cells were washed and samples acquired on a FACSymphony (BD Biosciences) at NIH. FACS data were analyzed using FlowJo 10 (BD Biosciences, FlowJo, LLC). 
The surgically-resected human lung tissues samples were weighed and manually aliquoted into replicates using sterile scissors inside a sterile $50 \mathrm{ml}$ tube. Aliquots were transferred into Trizol for RNA isolation or subjected to single cell isolation. Briefly, sample replicates for single cell isolation were digested with $100 \mathrm{U} / \mathrm{ml}$ of Collagenase IV (Sigma), $50 \mathrm{U} / \mathrm{ml}$ of Benzonase (Sigma) in $8 \mathrm{ml}$ of incomplete RPMI-1640 medium (without fetal bovine serum) for $45 \mathrm{~min}$ at $37^{\circ} \mathrm{C}$ in the shaker. Cells were then filtered through $100 \mu \mathrm{m}$ cell strainers (Miltenyi), and the remainder of tissue pieces were gently squashed by syringe and washed with $4 \mathrm{ml}$ ice-cold PBS containing $50 \%$ FBS to stop digestion. Next, the cell suspension was centrifuged and subjected to Debris Removal Solution (Miltenyi) according to the manufacturer's protocol. Samples were stained with surface antibodycocktails for $20 \mathrm{~min}$ at $4^{\circ} \mathrm{C}$, followed by fixable live/dead cell stain (Molecular Probes-Invitrogen). Cells were then fixed and permeabilized (eBioscience Transcription Factor Staining Buffer Kit) for at least $1 \mathrm{hr}$ followed by intracellular antigen staining for $30 \mathrm{~min}$ at $4^{\circ} \mathrm{C}$. Cells were washed and samples acquired on a LSRFortessa (BD Biosciences) at SPHCC. FACS data were analyzed using FlowJo 10 (BD Biosciences, FlowJo, LLC).

\section{Flow cytometry reagents}

Fluorochrome-labeled antibodies against mouse, human or rhesus macaque antigens used for flow cytometric analysis are listed below: I -A/I-E (clone M5/114.15.2), Ly6G (1A8), CD11c (HL3 and N418), CD45.2 (104), TCRb (H57-597), NK1.1 (PK136), CD11b (M1/70), CD45 (30-F11), CD68 (FA-11), Ly6C (AL-21 and HK1.4), Siglec-F (E50-2440), CD193 (J073E5), Arg1 (A1exF5), NOS2 (CXNFT), CD4 (L3T4), CD8a (53-6.7), Foxp3 (FJK-16s), IRF8 (V3GYWCH), XCR1 (ZET), CD193 (5E8.4), EPX (AHE-1), CD62L (MEL-14), CD14 (M5E2), CD11c (3.9), CD45 (D0581283), CD66abce (TET2), CD15 (HI98), CD68 (Y1/82A), HLA-DR (L243), CD16 (3G8), CD45 (2D1), CD193 (83101), CD66b (G10F5), CD62L (REG-56), CD206 (15-2.2), CD123 (6H6) and Siglec-8 (7C9). ESAT- ${ }_{1-17}$ or TB10.4-11 tetramers were produced by the NIAID tetramer core facility (Emory University). 


\section{Statistical analyses}

The statistical significance of differences between data groups were calculated using GraphPad Prism 8 as indicated in the figure legends. The Mann-Whitney test, the Wilcoxon matched pairs test (paired samples) were used for comparison of group means, Log-rank Mantel-Cox test for survival data and Spearman correlation test unless otherwise indicated in the figure or figure legends.

\section{Description of supplemental material}

Fig. S1 shows additional clinical data and study designs Fig. S2 human and nonhuman primate granuloma structure with eosinophil distribution and EPX staining specificity Fig. S3 shows depletion efficiency in eosinophil deficient mouse strains after Mtb infection, additional transcriptional and immunological parameters assessed in $\Delta \mathrm{db} \mid \mathrm{Gata}$ and increased bacterial loads in B6 PHIL mice.

\section{REFERENCES}

Abate, E., M. Belayneh, J. Idh, E. Diro, D. Elias, S. Britton, A. Aseffa, O. Stendahl, and T. Schon. 2015. Asymptomatic Helminth Infection in Active Tuberculosis Is Associated with Increased Regulatory and Th-2 Responses and a Lower Sputum Smear Positivity. PLoS Negl Trop Dis 9:e0003994.

Acharya, K.R., and S.J. Ackerman. 2014. Eosinophil granule proteins: form and function. J Biol Chem 289:17406-17415.

Anderson, K.G., K. Mayer-Barber, H. Sung, L. Beura, B.R. James, J.J. Taylor, L. Qunaj, T.S. Griffith, V. Vezys, D.L. Barber, and D. Masopust. 2014. Intravascular staining for discrimination of vascular and tissue leukocytes. Nat Protoc 9:209-222.

Arnold, I.C., M. Artola-Boran, P. Tallon de Lara, A. Kyburz, C. Taube, K. Ottemann, M. van den Broek, S. Yousefi, H.U. Simon, and A. Muller. 2018. Eosinophils suppress Th1 responses and restrict bacterially induced gastrointestinal inflammation. J Exp Med 215:2055-2072.

Babu, S., and T.B. Nutman. 2016. Helminth-Tuberculosis Co-infection: An Immunologic Perspective. Trends Immunol 37:597-607.

Basaraba, R.J., and R.L. Hunter. 2017. Pathology of Tuberculosis: How the Pathology of Human Tuberculosis Informs and Directs Animal Models. Microbiol Spectr 5:

Blauenfeldt, T., L. Petrone, F. Del Nonno, A. Baiocchini, L. Falasca, T. Chiacchio, V. Bondet, V. Vanini, F. Palmieri, G. Galluccio, A. Casrouge, J. Eugen-Olsen, M.L. Albert, D. 
Goletti, D. Duffy, and M. Ruhwald. 2018. Interplay of DDP4 and IP-10 as a Potential Mechanism for Cell Recruitment to Tuberculosis Lesions. Front Immunol 9:1456.

Bolger, A.M., M. Lohse, and B. Usadel. 2014. Trimmomatic: a flexible trimmer for Illumina sequence data. Bioinformatics 30:2114-2120.

Borelli, V., F. Vita, S. Shankar, M.R. Soranzo, E. Banfi, G. Scialino, C. Brochetta, and G. Zabucchi. 2003. Human eosinophil peroxidase induces surface alteration, killing, and lysis of Mycobacterium tuberculosis. Infect Immun 71:605-613.

Bozkurt, T.E. 2019. Endocannabinoid System in the Airways. Molecules 24:

Chesne, J., V. Cardoso, and H. Veiga-Fernandes. 2019. Neuro-immune regulation of mucosal physiology. Mucosal Immunol 12:10-20.

Cohen, S.B., B.H. Gern, J.L. Delahaye, K.N. Adams, C.R. Plumlee, J.K. Winkler, D.R. Sherman, M.Y. Gerner, and K.B. Urdahl. 2018. Alveolar Macrophages Provide an Early Mycobacterium tuberculosis Niche and Initiate Dissemination. Cell Host Microbe

Cooper, A.M., D.K. Dalton, T.A. Stewart, J.P. Griffin, D.G. Russell, and I.M. Orme. 1993. Disseminated tuberculosis in interferon gamma gene-disrupted mice. Journal of Experimental Medicine 178:2243-2247.

Costello, R.W., B.H. Schofield, G.M. Kephart, G.J. Gleich, D.B. Jacoby, and A.D. Fryer. 1997. Localization of eosinophils to airway nerves and effect on neuronal M2 muscarinic receptor function. Am J Physiol 273:L93-103.

Cronan, M.R., E.J. Hughes, W.J. Brewer, G. Viswanathan, E.G. Hunt, B. Singh, S. Mehra, S.H. Oehlers, S.G. Gregory, D. Kaushal, and D.M. Tobin. 2021. A non-canonical type 2 immune response coordinates tuberculous granuloma formation and epithelialization. Cell 184:1757-+.

Dobin, A., C.A. Davis, F. Schlesinger, J. Drenkow, C. Zaleski, S. Jha, P. Batut, M. Chaisson, and T.R. Gingeras. 2013. STAR: ultrafast universal RNA-seq aligner. Bioinformatics 29:1521.

Drake, M.G., K.M. Lebold, Q.R. Roth-Carter, A.B. Pincus, E.D. Blum, B.J. Proskocil, D.B. Jacoby, A.D. Fryer, and Z. Nie. 2018a. Eosinophil and airway nerve interactions in asthma. J Leukoc Biol 104:61-67.

Drake, M.G., G.D. Scott, E.D. Blum, K.M. Lebold, Z. Nie, J.J. Lee, A.D. Fryer, R.W. Costello, and D.B. Jacoby. 2018b. Eosinophils increase airway sensory nerve density in mice and in human asthma. Sci Transl Med 10:

Du Bruyn, E., S. Ruzive, C.S. Lindestam Arlehamn, A. Sette, A. Sher, D.L. Barber, R.J. Wilkinson, and C. Riou. 2020. Mycobacterium tuberculosis-specific CD4 T cells expressing CD153 inversely associate with bacterial load and disease severity in human tuberculosis. Mucosal Immunol

Ernst, J.D. 2012. The immunological life cycle of tuberculosis. Nat Rev Immunol 12:581-591.

Flynn, J.L., J. Chan, K.J. Triebold, D.K. Dalton, T.A. Stewart, and B.R. Bloom. 1993. An essential role for interferon gamma in resistance to Mycobacterium tuberculosis infection. Journal of Experimental Medicine 178:2249-2254.

Flynn, J.L., and J.D. Ernst. 2000. Immune responses in tuberculosis. Curr Opin Immunol 12:432436.

Flynn, J.L., H.P. Gideon, J.T. Mattila, and P.L. Lin. 2015. Immunology studies in non-human primate models of tuberculosis. Immunol Rev 264:60-73.

Fryer, A.D., and M. Wills-Karp. 1991. Dysfunction of M2-muscarinic receptors in pulmonary parasympathetic nerves after antigen challenge. J Appl Physiol (1985) 71:2255-2261. 
Huang, L., E.V. Nazarova, S. Tan, Y. Liu, and D.G. Russell. 2018. Growth of Mycobacterium tuberculosis in vivo segregates with host macrophage metabolism and ontogeny. $J$ Exp Med 215:1135-1152.

Kingham, P.J., W.G. McLean, D.A. Sawatzky, M.T. Walsh, and R.W. Costello. 2002. Adhesiondependent interactions between eosinophils and cholinergic nerves. Am J Physiol Lung Cell Mol Physiol 282:L1229-1238.

Kirman, J., Z. Zakaria, K. McCoy, B. Delahunt, and G. Le Gros. 2000. Role of eosinophils in the pathogenesis of Mycobacterium bovis BCG infection in gamma interferon receptordeficient mice. Infection and Immunity 68:2976-2978.

Klion, A.D., S.J. Ackerman, and B.S. Bochner. 2020. Contributions of Eosinophils to Human Health and Disease. Annu Rev Pathol 15:179-209.

Klion, A.D., and T.B. Nutman. 2004. The role of eosinophils in host defense against helminth parasites. J Allergy Clin Immunol 113:30-37.

Krishack, P.A., T.J. Louviere, T.S. Decker, T.G. Kuzel, J.A. Greenberg, D.F. Camacho, C.L. Hrusch, A.I. Sperling, and P.A. Verhoef. 2019. Protection against Staphylococcus aureus bacteremia-induced mortality depends on ILC2s and eosinophils. JCI Insight 4:

Lasco, T.M., O.C. Turner, L. Cassone, I. Sugawara, H. Yamada, D.N. McMurray, and I.M. Orme. 2004. Rapid accumulation of eosinophils in lung lesions in guinea pigs infected with Mycobacterium tuberculosis. Infect Immun 72:1147-1149.

Lee, J., S. Boyce, J. Powers, C. Baer, C.M. Sassetti, and S.M. Behar. 2020. CD11cHi monocytederived macrophages are a major cellular compartment infected by Mycobacterium tuberculosis. PLoS Pathog 16:e1008621.

Lee, J.J., D. Dimina, M.P. Macias, S.I. Ochkur, M.P. McGarry, K.R. O'Neill, C. Protheroe, R. Pero, T. Nguyen, S.A. Cormier, E. Lenkiewicz, D. Colbert, L. Rinaldi, S.J. Ackerman, C.G. Irvin, and N.A. Lee. 2004. Defining a link with asthma in mice congenitally deficient in eosinophils. Science 305:1773-1776.

Lee, J.J., E.A. Jacobsen, M.P. McGarry, R.P. Schleimer, and N.A. Lee. 2010. Eosinophils in health and disease: the LIAR hypothesis. Clin Exp Allergy 40:563-575.

Lilly, L.M., M. Scopel, M.P. Nelson, A.R. Burg, C.W. Dunaway, and C. Steele. 2014. Eosinophil deficiency compromises lung defense against Aspergillus fumigatus. Infect Immun 82:1315-1325.

Linch, S.N., A.M. Kelly, E.T. Danielson, R. Pero, J.J. Lee, and J.A. Gold. 2009. Mouse eosinophils possess potent antibacterial properties in vivo. Infect Immun 77:4976-4982.

Love, M.I., W. Huber, and S. Anders. 2014. Moderated estimation of fold change and dispersion for RNA-seq data with DESeq2. Genome Biol 15:550.

Lowe, D.M., A.K. Bandara, G.E. Packe, R.D. Barker, R.J. Wilkinson, C.J. Griffiths, and A.R. Martineau. 2013. Neutrophilia independently predicts death in tuberculosis. Eur Respir J 42:1752-1757.

Mayer-Barber, K.D., B.B. Andrade, S.D. Oland, E.P. Amaral, D.L. Barber, J. Gonzales, S.C. Derrick, R. Shi, N.P. Kumar, W. Wei, X. Yuan, G. Zhang, Y. Cai, S. Babu, M. Catalfamo, A.M. Salazar, L.E. Via, C.E. Barry, 3rd, and A. Sher. 2014. Host-directed therapy of tuberculosis based on interleukin-1 and type I interferon crosstalk. Nature 511:99-103.

Mayer-Barber, K.D., and A. Sher. 2015. Cytokine and lipid mediator networks in tuberculosis. Immunol Rev 264:264-275. 
O'Garra, A., P.S. Redford, F.W. McNab, C.I. Bloom, R.J. Wilkinson, and M.P. Berry. 2013. The immune response in tuberculosis. Annu Rev Immunol 31:475-527.

O'Shea, M.K., T.E. Fletcher, J. Muller, R. Tanner, M. Matsumiya, J.W. Bailey, J. Jones, S.G. Smith, G. Koh, W.G. Horsnell, N.J. Beeching, J. Dunbar, D. Wilson, A.F. Cunningham, and H. McShane. 2018. Human Hookworm Infection Enhances Mycobacterial Growth Inhibition and Associates With Reduced Risk of Tuberculosis Infection. Front Immunol 9:2893.

Parikka, M., M.M. Hammaren, S.K. Harjula, N.J. Halfpenny, K.E. Oksanen, M.J. Lahtinen, E.T. Pajula, A. Iivanainen, M. Pesu, and M. Ramet. 2012. Mycobacterium marinum causes a latent infection that can be reactivated by gamma irradiation in adult zebrafish. PLoS Pathog 8:e1002944.

Pavord, I.D., S. Korn, P. Howarth, E.R. Bleecker, R. Buhl, O.N. Keene, H. Ortega, and P. Chanez. 2012. Mepolizumab for severe eosinophilic asthma (DREAM): a multicentre, double-blind, placebo-controlled trial. Lancet 380:651-659.

Phipps, S., C.E. Lam, S. Mahalingam, M. Newhouse, R. Ramirez, H.F. Rosenberg, P.S. Foster, and K.I. Matthaei. 2007. Eosinophils contribute to innate antiviral immunity and promote clearance of respiratory syncytial virus. Blood 110:1578-1586.

Pisu, D., L. Huang, J.K. Grenier, and D.G. Russell. 2020. Dual RNA-Seq of Mtb-Infected Macrophages In Vivo Reveals Ontologically Distinct Host-Pathogen Interactions. Cell Rep 30:335-350 e334.

Rafi, W., R. Ribeiro-Rodrigues, J.J. Ellner, and P. Salgame. 2012. 'Coinfection-helminthes and tuberculosis'. Curr Opin HIV AIDS 7:239-244.

Rosenberg, H.F., K.D. Dyer, and P.S. Foster. 2013. Eosinophils: changing perspectives in health and disease. Nat Rev Immunol 13:9-22.

Rothchild, A.C., G.S. Olson, J. Nemeth, L.M. Amon, D. Mai, E.S. Gold, A.H. Diercks, and A. Aderem. 2019. Alveolar macrophages generate a noncanonical NRF2-driven transcriptional response to Mycobacterium tuberculosis in vivo. Sci Immunol 4:

Rothenberg, M.E., A.D. Klion, F.E. Roufosse, J.E. Kahn, P.F. Weller, H.U. Simon, L.B. Schwartz, L.J. Rosenwasser, J. Ring, E.F. Griffin, A.E. Haig, P.I. Frewer, J.M. Parkin, G.J. Gleich, and H.E.S.S.G. Mepolizumab. 2008. Treatment of patients with the hypereosinophilic syndrome with mepolizumab. N Engl J Med 358:1215-1228.

Ruhl, C.R., B.L. Pasko, H.S. Khan, L.M. Kindt, C.E. Stamm, L.H. Franco, C.C. Hsia, M. Zhou, C.R. Davis, T. Qin, L. Gautron, M.D. Burton, G.L. Mejia, D.K. Naik, G. Dussor, T.J. Price, and M.U. Shiloh. 2020. Mycobacterium tuberculosis Sulfolipid-1 Activates Nociceptive Neurons and Induces Cough. Cell 181:293-305 e211.

Sallin, M.A., K.D. Kauffman, C. Riou, E. Du Bruyn, T.W. Foreman, S. Sakai, S.G. Hoft, T.G. Myers, P.J. Gardina, A. Sher, R. Moore, T. Wilder-Kofie, I.N. Moore, A. Sette, C.S. Lindestam Arlehamn, R.J. Wilkinson, and D.L. Barber. 2018. Host resistance to pulmonary Mycobacterium tuberculosis infection requires CD153 expression. Nat Microbiol 3:1198-1205.

Shah, K., A. Ignacio, K.D. McCoy, and N.L. Harris. 2020. The emerging roles of eosinophils in mucosal homeostasis. Mucosal Immunol 13:574-583.

Shamri, R., J.J. Xenakis, and L.A. Spencer. 2011. Eosinophils in innate immunity: an evolving story. Cell Tissue Res 343:57-83. 
Simon, H.U., S. Yousefi, N. Germic, I.C. Arnold, A. Haczku, A.V. Karaulov, D. Simon, and H.F. Rosenberg. 2020. The Cellular Functions of Eosinophils: Collegium Internationale Allergologicum (CIA) Update 2020. Int Arch Allergy Immunol 181:11-23.

Singhania, A., C.M. Graham, L. Gabrysova, L. Moreira-Teixeira, E. Stavropoulos, J.M. Pitt, P. Chakravarty, A. Warnatsch, W.J. Branchett, L. Conejero, J.W. Lin, S. Davidson, M.S. Wilson, G. Bancroft, J. Langhorne, E. Frickel, A.K. Sesay, S.L. Priestnall, E. Herbert, M. Ioannou, Q. Wang, I.R. Humphreys, J. Dodd, P.J.M. Openshaw, K.D. Mayer-Barber, D. Jankovic, A. Sher, C.M. Lloyd, N. Baldwin, D. Chaussabel, V. Papayannopoulos, A. Wack, J.F. Banchereau, V.M. Pascual, and A. O'Garra. 2019. Transcriptional profiling unveils type I and II interferon networks in blood and tissues across diseases. Nat Commun 10:2887.

Soneson, C., M.I. Love, and M.D. Robinson. 2015. Differential analyses for RNA-seq: transcript-level estimates improve gene-level inferences. F1000Res 4:1521.

Straumann, A., S. Conus, P. Grzonka, H. Kita, G. Kephart, C. Bussmann, C. Beglinger, D.A. Smith, J. Patel, M. Byrne, and H.U. Simon. 2010. Anti-interleukin-5 antibody treatment (mepolizumab) in active eosinophilic oesophagitis: a randomised, placebo-controlled, double-blind trial. Gut 59:21-30.

Swaim, L., L. Connolly, H. Volkman, O. Humbert, D. Born, and L. Ramakrishnan. 2006. Mycobacterium marinum infection of adult zebrafish causes caseating granulomatous tuberculosis and is moderated by adaptive immunity. Infection and Immunity 74:61086117.

Takaki, K., J.M. Davis, K. Winglee, and L. Ramakrishnan. 2013. Evaluation of the pathogenesis and treatment of Mycobacterium marinum infection in zebrafish. Nat Protoc 8:11141124.

Trauner, A., Q. Liu, L.E. Via, X. Liu, X. Ruan, L. Liang, H. Shi, Y. Chen, Z. Wang, R. Liang, W. Zhang, W. Wei, J. Gao, G. Sun, D. Brites, K. England, G. Zhang, S. Gagneux, C.E. Barry, 3rd, and Q. Gao. 2017. The within-host population dynamics of Mycobacterium tuberculosis vary with treatment efficacy. Genome Biol 18:71.

Travers, J., and M.E. Rothenberg. 2015. Eosinophils in mucosal immune responses. Mucosal Immunol 8:464-475.

van Soelen, N., A.M. Mandalakas, H.L. Kirchner, G. Walzl, H.M. Grewal, M. Jacobsen, and A.C. Hesseling. 2012. Effect of Ascaris Lumbricoides specific IgE on tuberculin skin test responses in children in a high-burden setting: a cross-sectional community-based study. BMC Infect Dis 12:211.

Veiga-Fernandes, H., and D. Mucida. 2016. Neuro-Immune Interactions at Barrier Surfaces. Cell 165:801-811.

Vijayan, V.K., A.M. Reetha, M.S. Jawahar, K. Sankaran, and R. Prabhakar. 1992. Pulmonary eosinophilia in pulmonary tuberculosis. Chest 101:1708-1709.

Vinhaes, C.L., D. Oliveira-de-Souza, P.S. Silveira-Mattos, B. Nogueira, R. Shi, W. Wei, X. Yuan, G. Zhang, Y. Cai, C.E. Barry, 3rd, L.E. Via, K.F. Fukutani, B.B. Andrade, and K.D. Mayer-Barber. 2019. Changes in inflammatory protein and lipid mediator profiles persist after antitubercular treatment of pulmonary and extrapulmonary tuberculosis: A prospective cohort study. Cytokine 123:154759.

WHO. 2020. Global Tuberculosis Report 2020. https://apps.who.int/iris/bitstream/handle/10665/336069/9789240013131-eng.pdf 
Wolf, A.J., B. Linas, G.J. Trevejo-Nunez, E. Kincaid, T. Tamura, K. Takatsu, and J.D. Ernst. 2007. Mycobacterium tuberculosis infects dendritic cells with high frequency and impairs their function in vivo. Journal of Immunology 179:2509-2519.

Yousefi, S., J.A. Gold, N. Andina, J.J. Lee, A.M. Kelly, E. Kozlowski, I. Schmid, A. Straumann, J. Reichenbach, G.J. Gleich, and H.U. Simon. 2008. Catapult-like release of mitochondrial DNA by eosinophils contributes to antibacterial defense. Nat Med 14:949953.

Yu, C., A.B. Cantor, H. Yang, C. Browne, R.A. Wells, Y. Fujiwara, and S.H. Orkin. 2002. Targeted deletion of a high-affinity GATA-binding site in the GATA-1 promoter leads to selective loss of the eosinophil lineage in vivo. J Exp Med 195:1387-1395.

Yu, G., and Q.Y. He. 2016. ReactomePA: an R/Bioconductor package for reactome pathway analysis and visualization. Mol Biosyst 12:477-479.

Yu, G., L.G. Wang, Y. Han, and Q.Y. He. 2012. clusterProfiler: an R package for comparing biological themes among gene clusters. OMICS 16:284-287. 
bioRxiv preprint doi: https://doi.org/10.1101/2021.05.29.446277; this version posted May 31, 2021. The copyright holder for this preprint (which was not certified by peer review) is the author/funder. This article is a US Government work. It is not subject to copyright under 17 USC 105 and is also made available for use under a CCO license. Bohrer et al. Figure 1
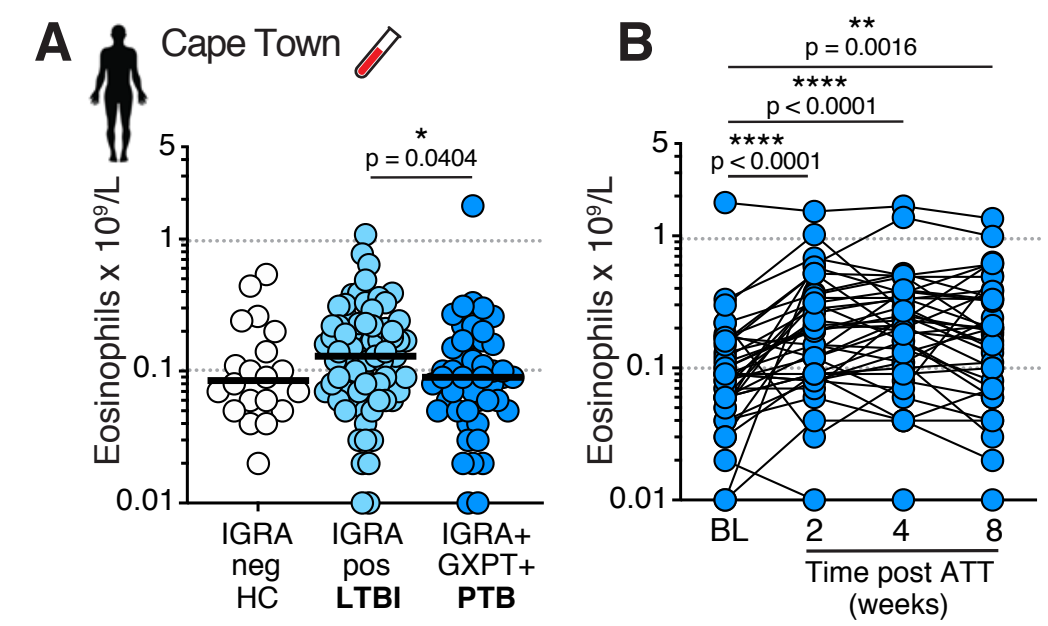

C Shanghai

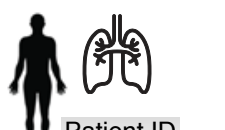

Gated on Live CD45+

Patient ID

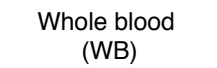

Re Resected human lung tissue

- ID\#1 ID\#2

- ID\#3

ID\#4

ID\#5
ID\#6

ID\#7

ID\#8
ID\#9

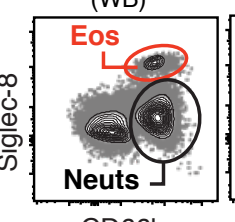
healthy region Fibrotic region Granuloma/nodule

CD66b

$\bigcirc$ Eosinophils (eos) $\triangle$ Neutrophils (neuts) $\square$ Eos/Neuts ratio (E/N)

D

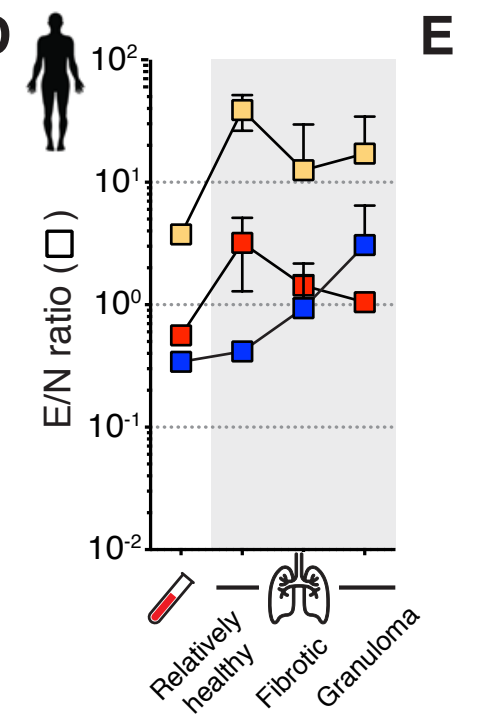

Relatively Fibrotic

(⿻) healthy region region

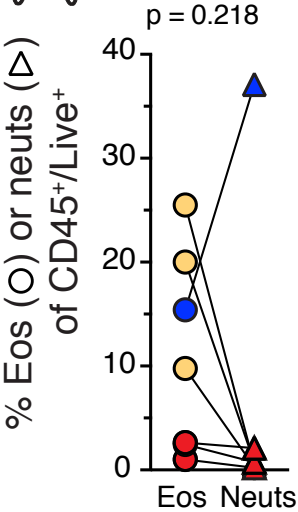

Eos Neuts
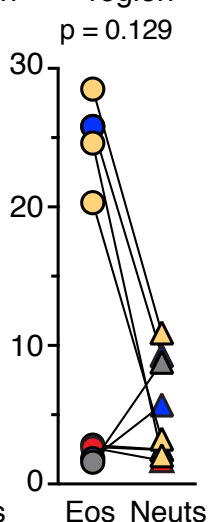

Granuloma/ F nodule $p=0.240$

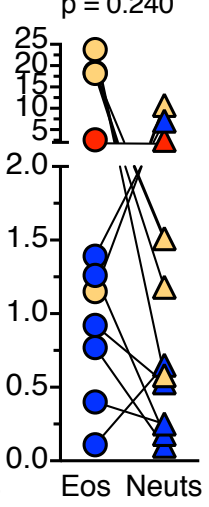

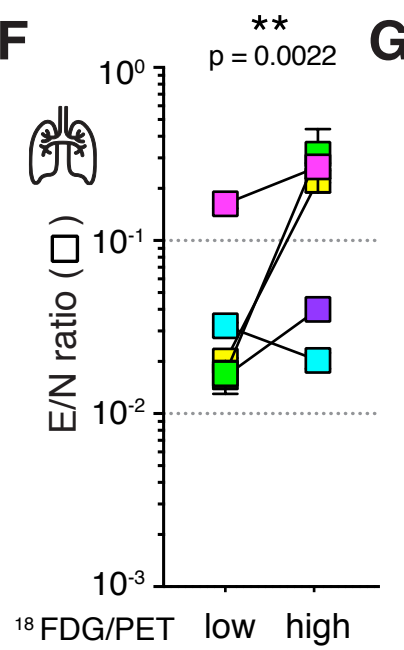

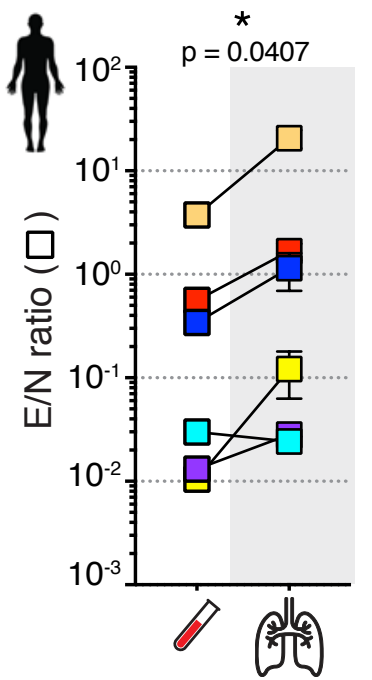


bioRxiv preprint doi: https://doi.org/10.1101/2021.05.29.446277; this version posted May 31, 2021. The copyright holder for this preprint (which was not certified by peer review) is the author/funder. This article is a US Government work. It is not subject to copyright under 17 USC 105 and is also made available for use under a CCO license.

\section{Bohrer et al. Figure 2}
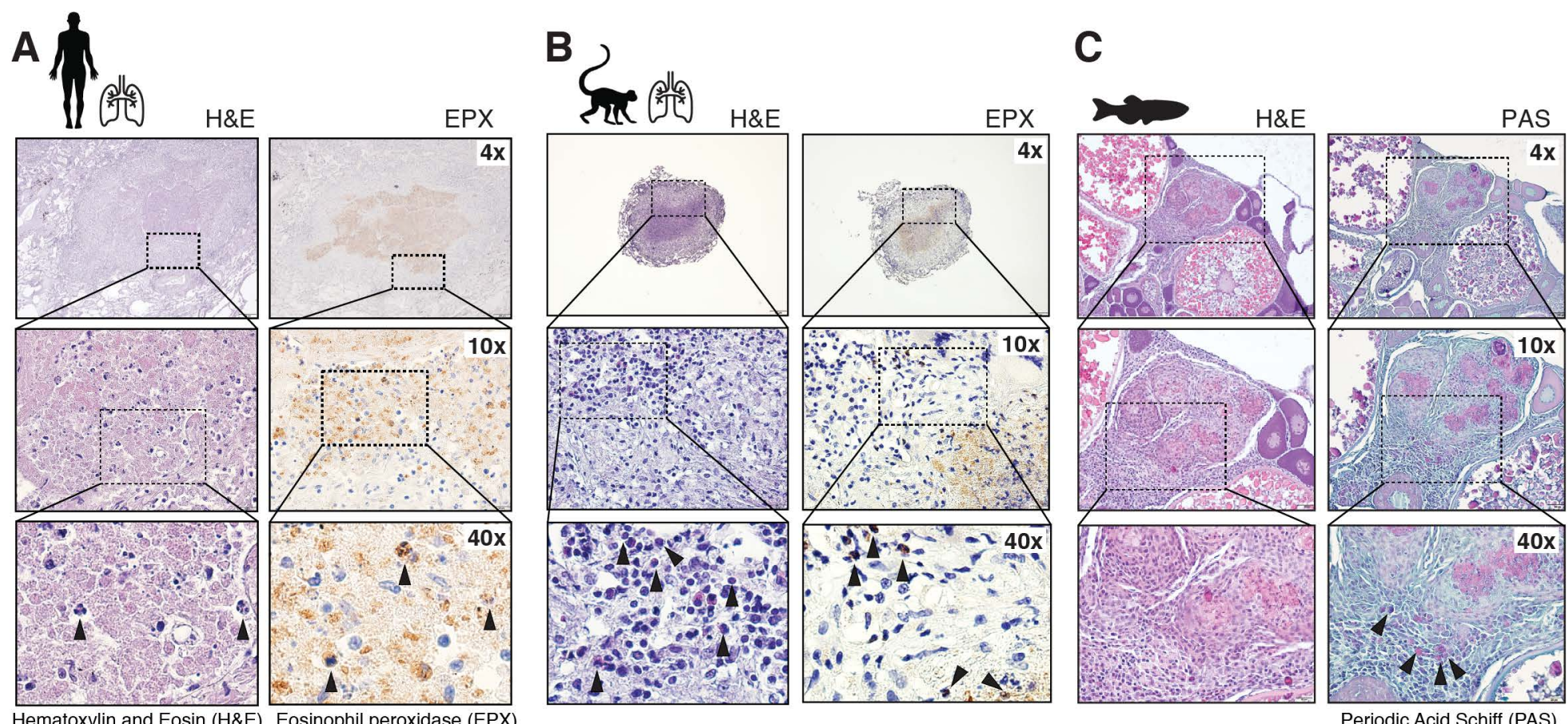

Periodic Acid Schiff (PAS) 


\section{Bohrer et al. Figure 3}

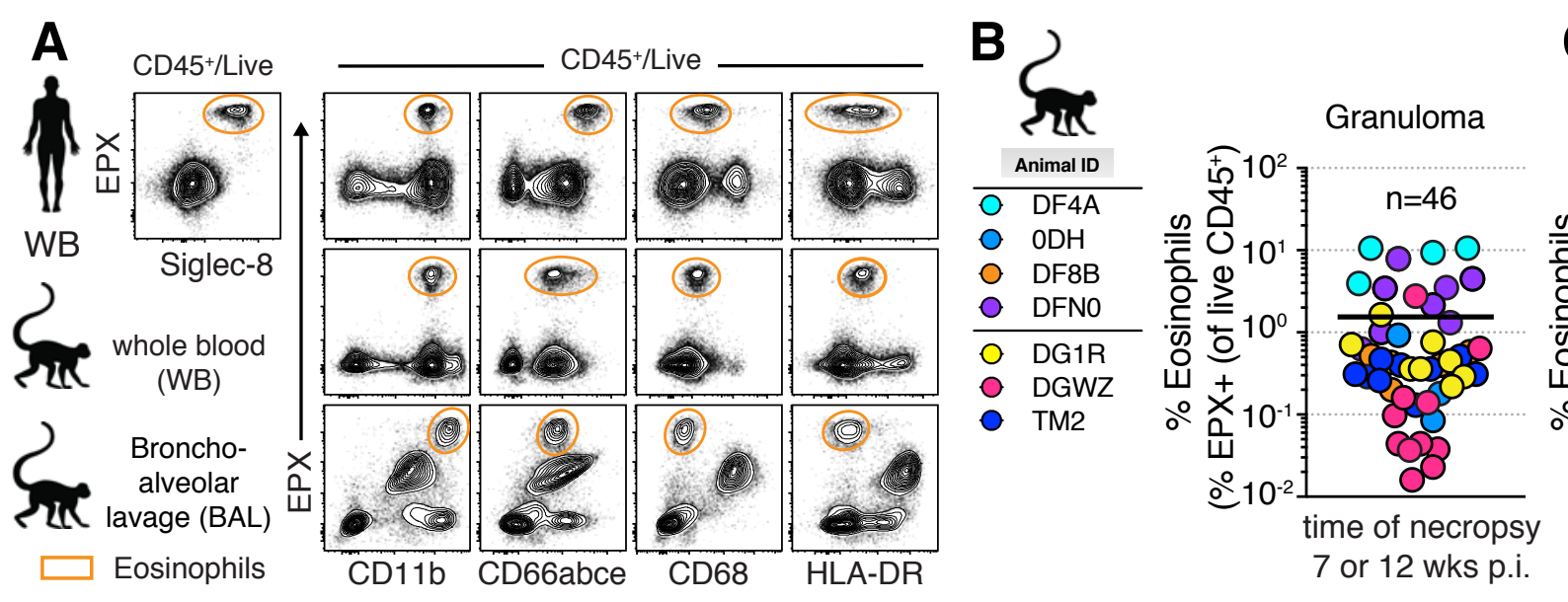

C

D

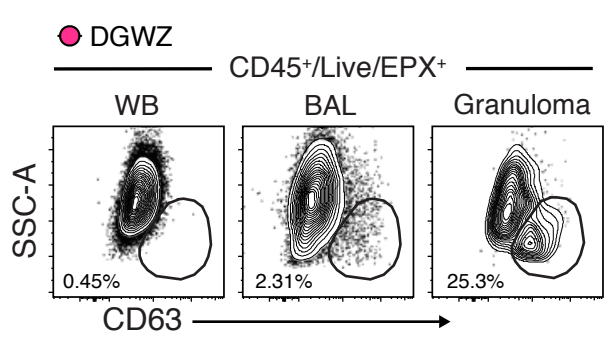

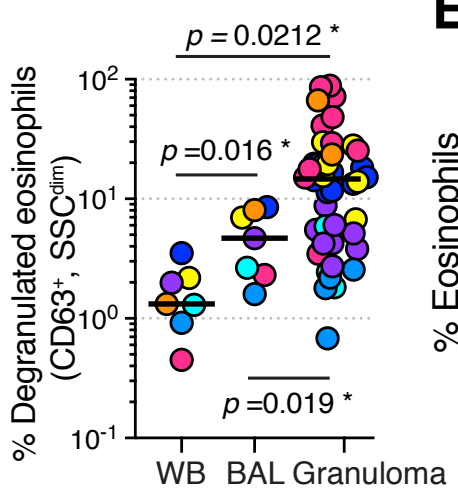

E

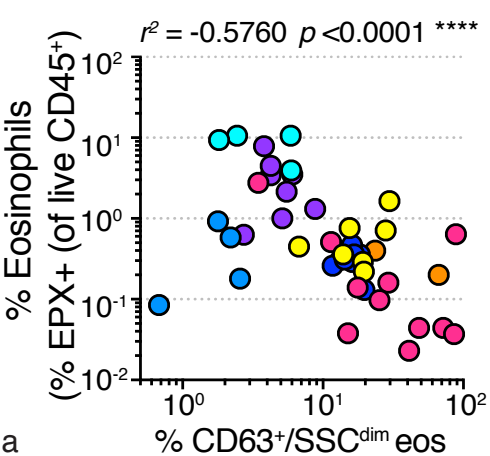

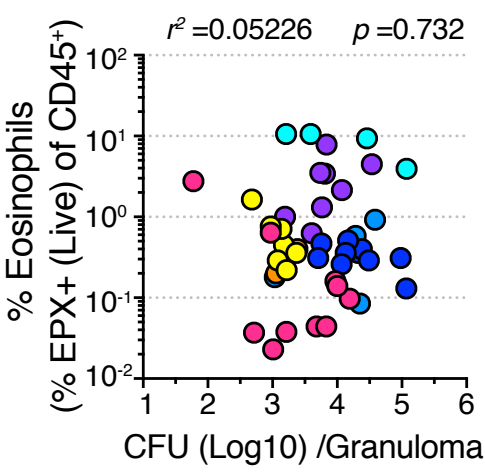

$\mathbf{F}$

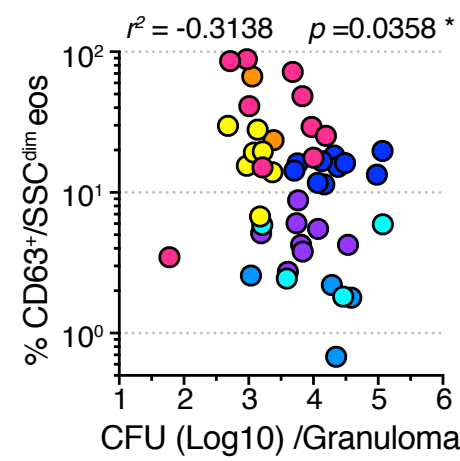


A

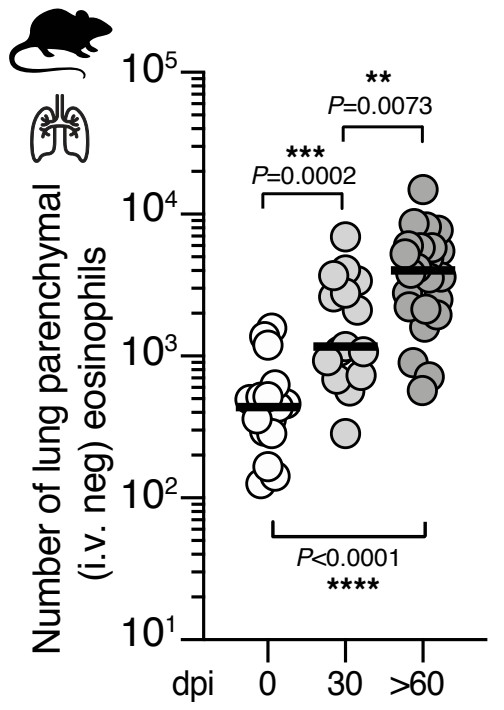

B
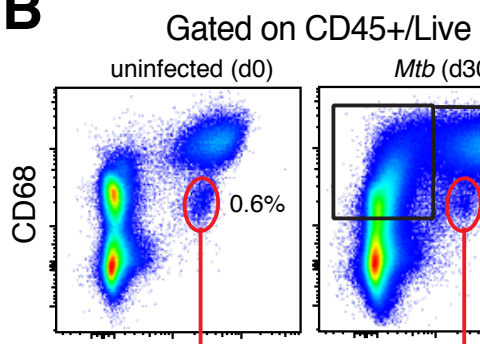

Siglec-Ft

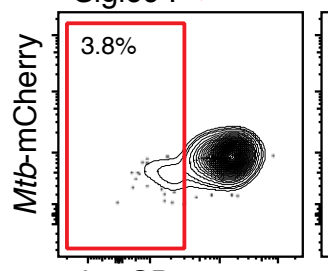

i.v. CD45
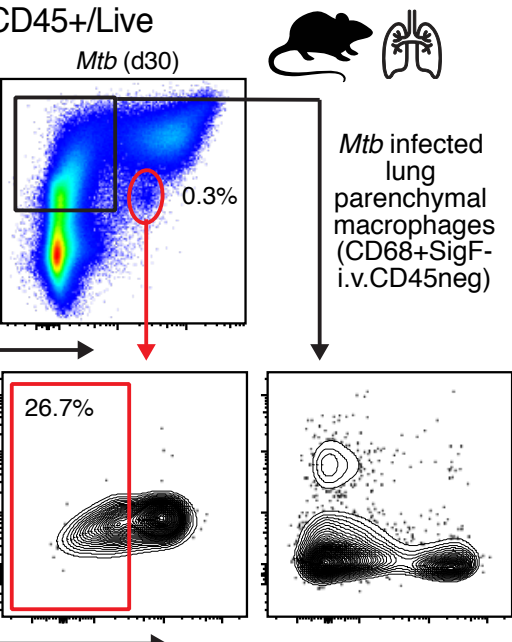

C
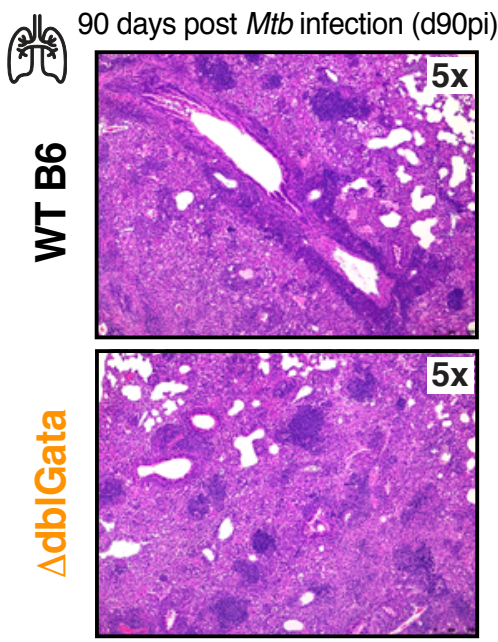

D DEG d90pi Mtb WT B6 vs. $\triangle \mathrm{dlblGata}$

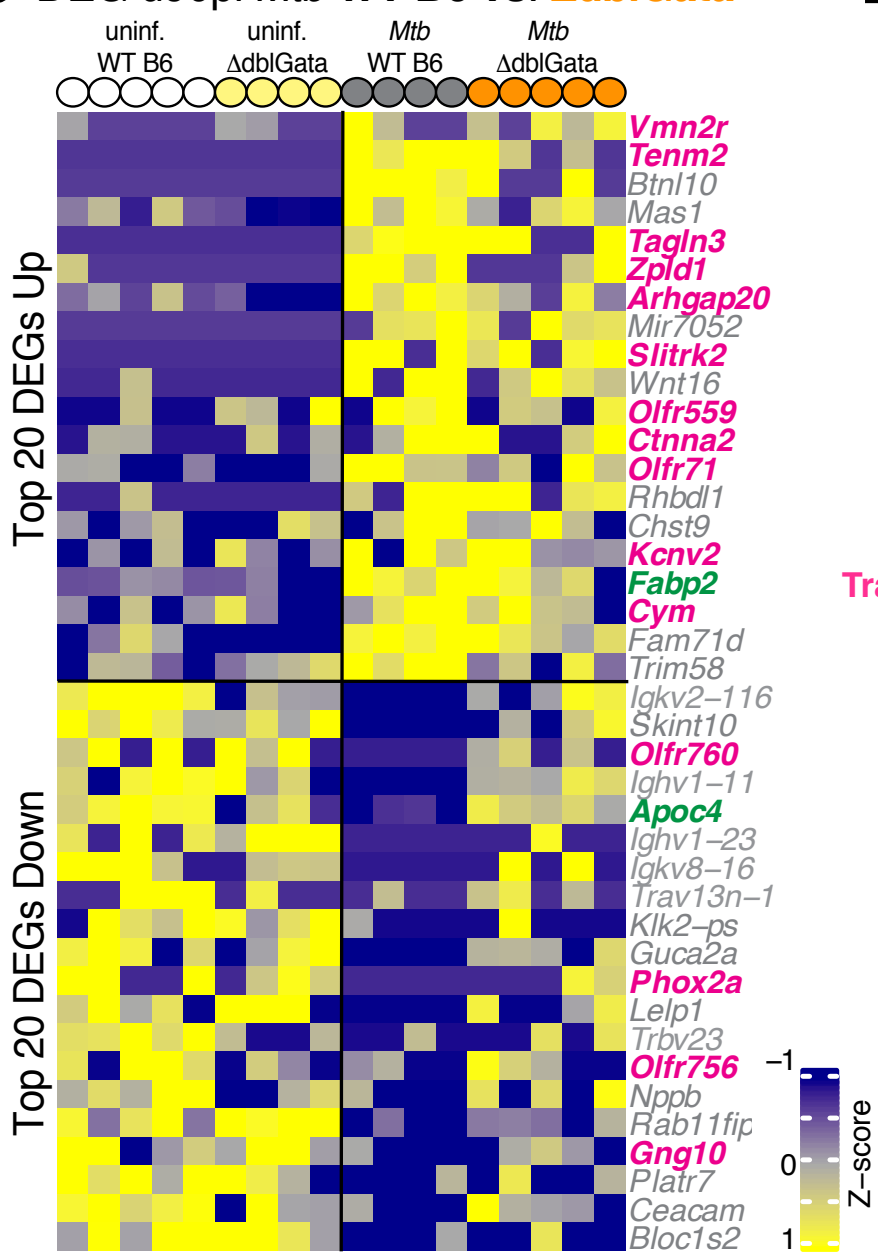

Neuronal-associated genes

Lipid and short chain fatty acid metabolism
E

\section{Reactome}

SLC-mediated transmembrane transport Transport of inorganic cations/anions and amino acids/oligopeptides Cytochrome P450 - arranged by substrate type Arachidonic acid metabolism Cell junction organization GABA receptor activationCell-cell junction organization Synthesis of Leukotrienes and Eoxins Eicosanoids Metabolism of Angiotensinogen to Angiotensins Trafficking of GluR2-containing AMPA receptors

KEGG

$$
p \text { range }(0.01-0.04)
$$

Retrograde endocannabinoid signaling Cushing syndrome Fluid shear stress and atherosclerosis Insulin secretion TGF-beta signaling pathway AMPK signaling pathway Insulin resistance Retinol metabolism Melanogenesis Chemical carcinogenesis Gastric acid secretion Metabolism of xenobiotics by cytochrome P450 Amphetamine addiction Cocaine addiction $p$ range $(0.01-0.04) 4$
Pathways down in $\Delta$ dblGata d90pi Mtb
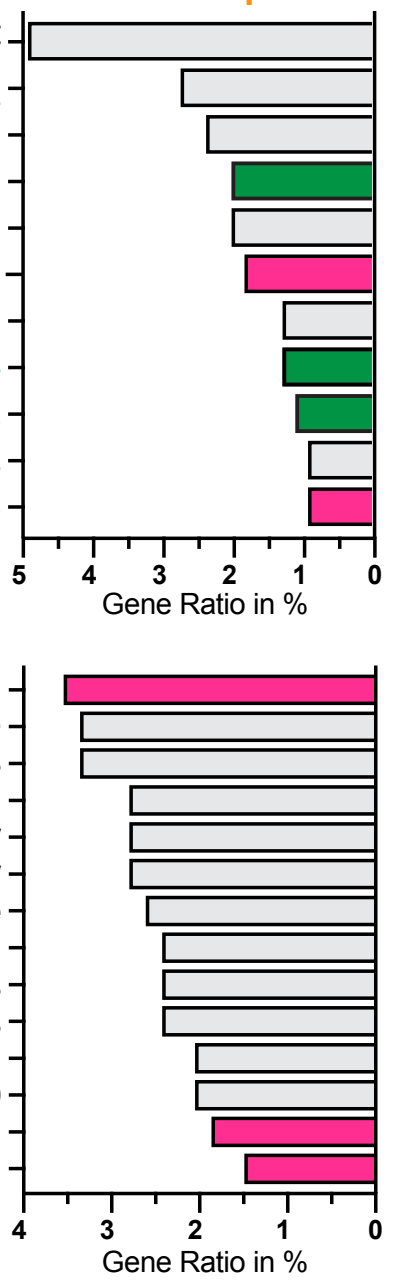
Bohrer et al. Figure 5

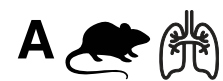

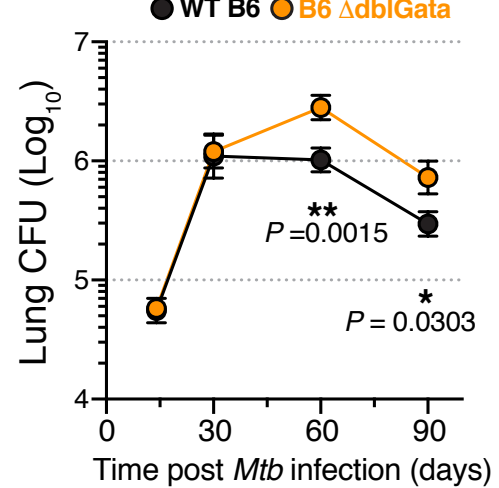

DE

略绝

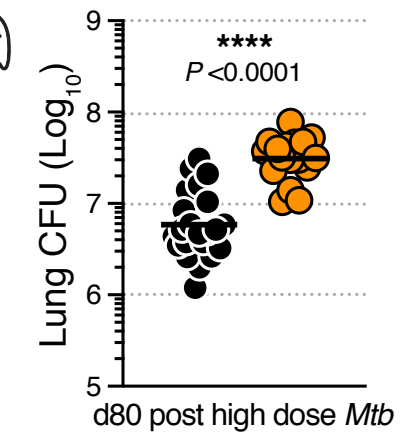

B

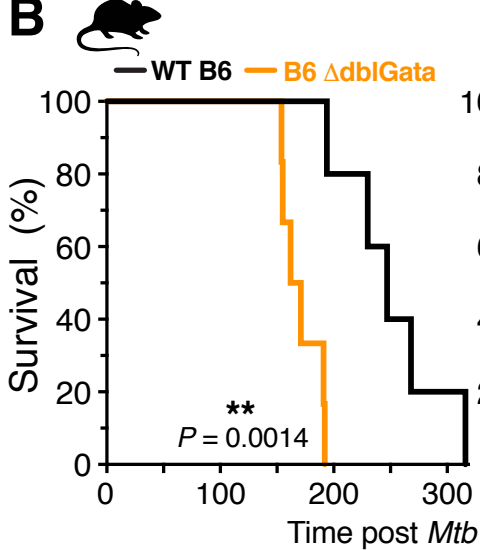

$\mathbf{E}$

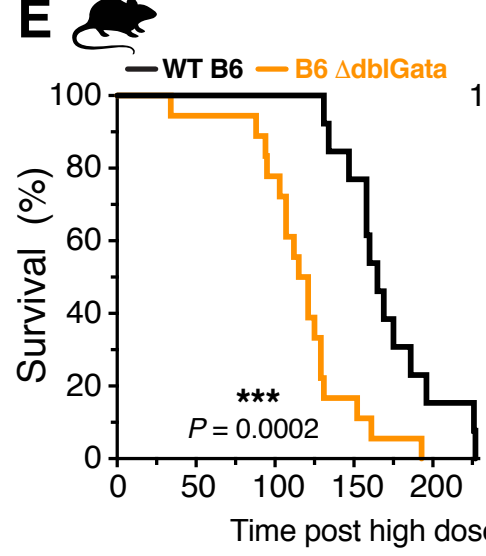

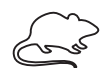

- Balb/C … Balb/C $\Delta$ dblGata
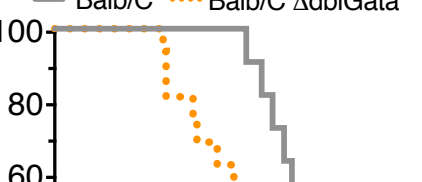

1

40. 20

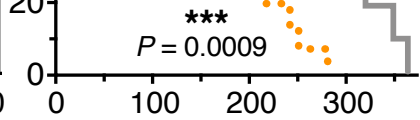

b infection (days)

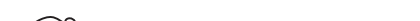

C

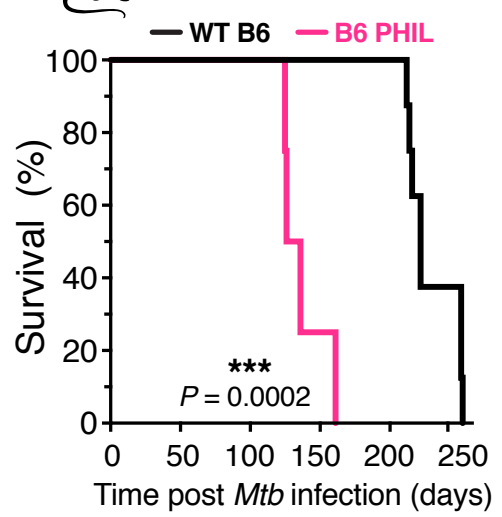

$F$

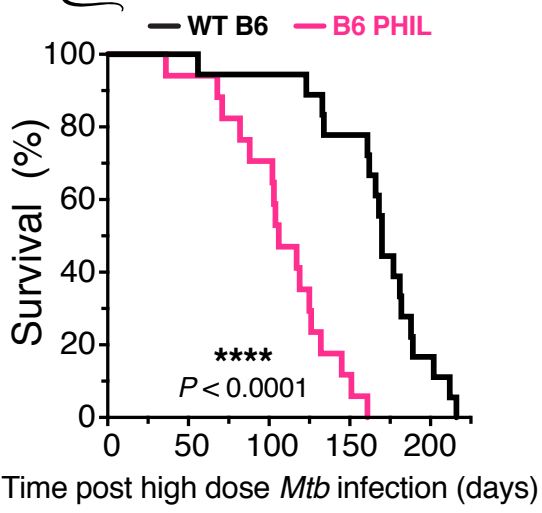


bioRxiv preprint doi: https://doi.org/10.1101/2021.05.29.446277; this version posted May 31, 2021. The copyright holder for this preprint (which was not certified by peer review) is the author/funder. This article is a US Government work. It is not subject to copyright under 17 USC 105 ảd is also made available for use undar accollicenss.

A

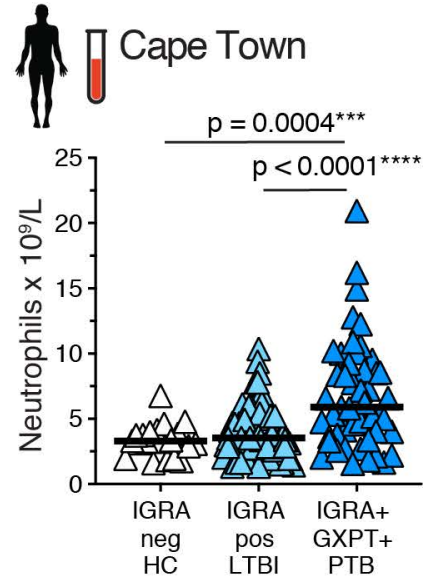

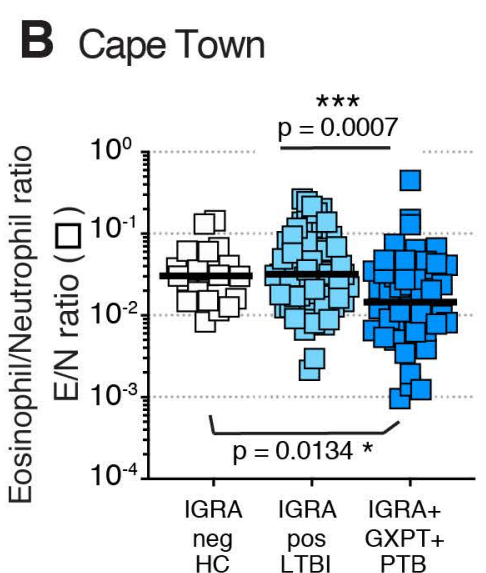
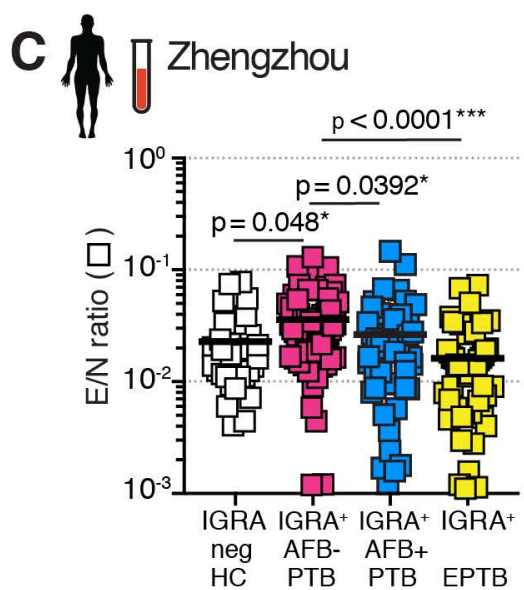

\section{Z Zhengzhou}

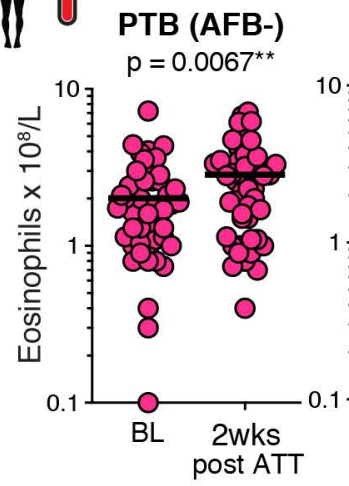

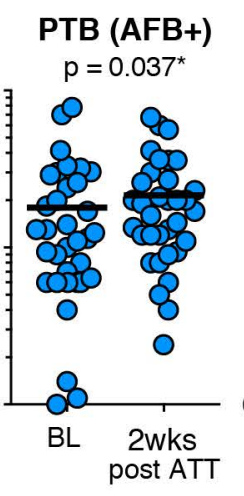

EPTB

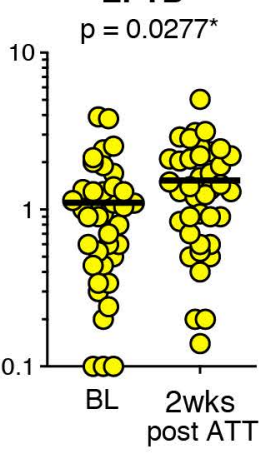

E \& Dondon

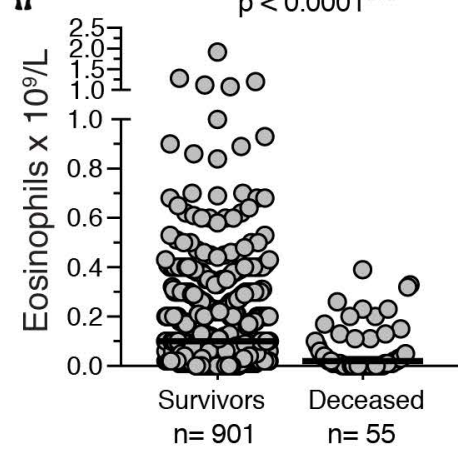

F

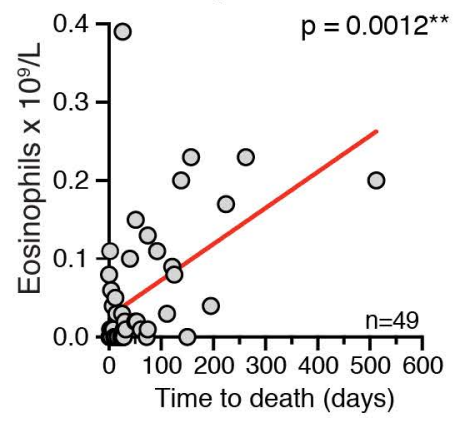

Time to death (days)
G Shanghai

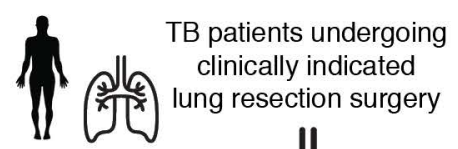

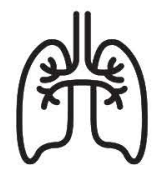

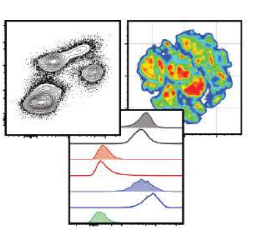

Same-day enzymatic single cell isolation and multi-parameter flow cytometry

macroscopic dissection based on presenting tissue pathologies, or in some cases (ID\#5-9) ${ }^{18}$ FDG-PET-CT signal
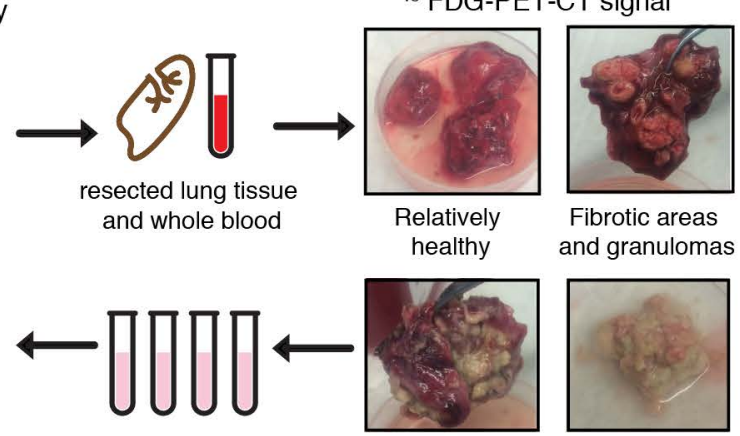

Caseum

$\mathrm{H}$ $\prod_{p=0.188} \begin{array}{ccc}p=0.844 & p=0.125 & p=0.359\end{array}$

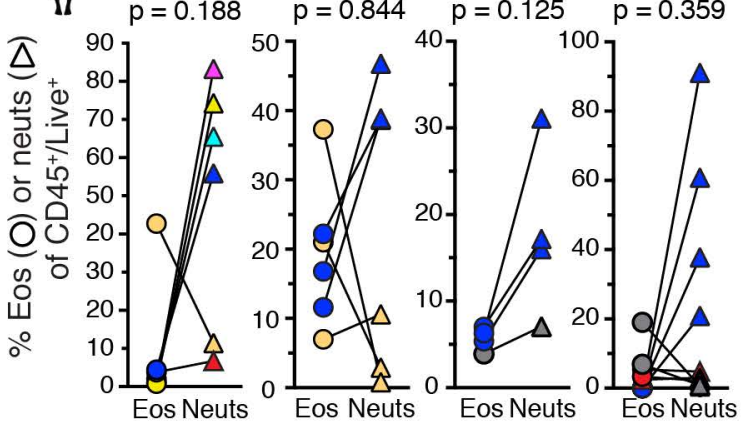

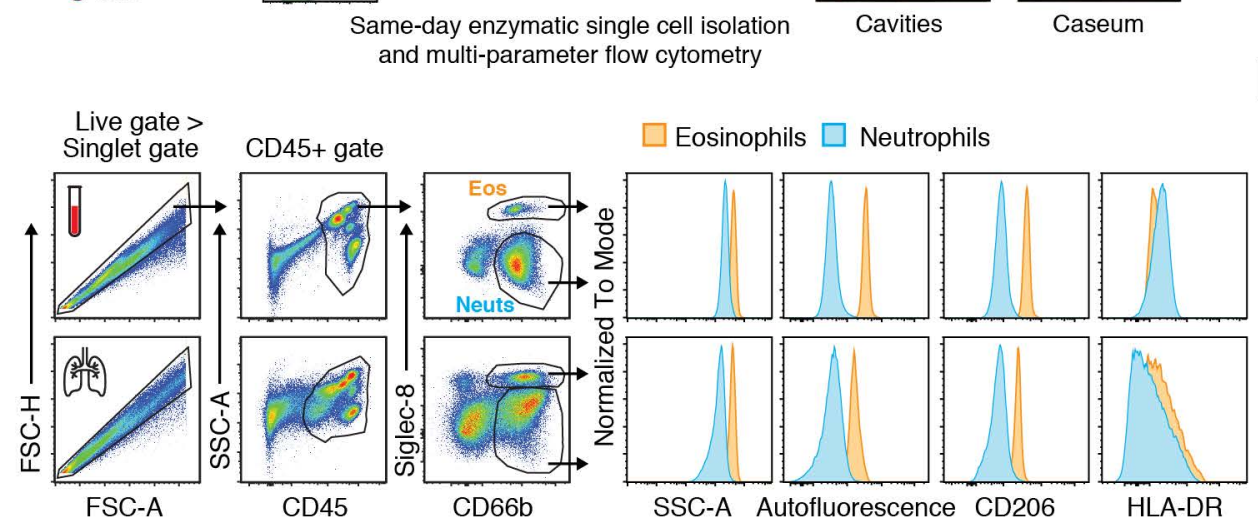
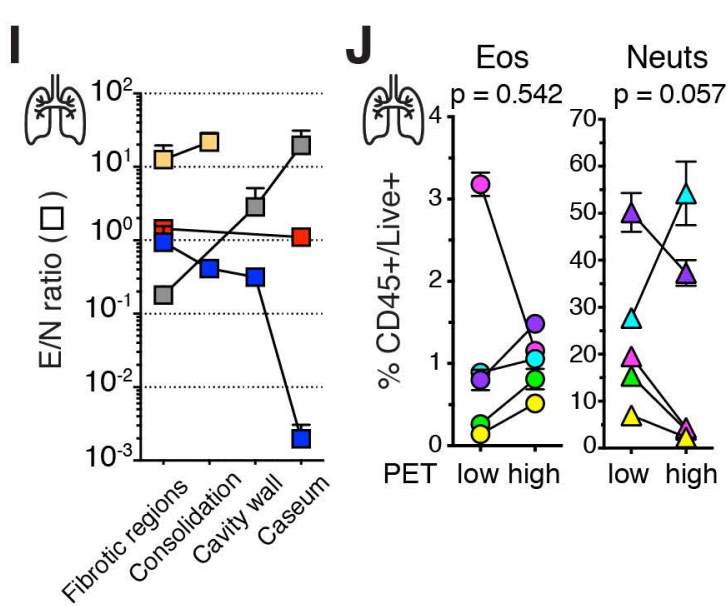
bioRxiv preprint doi: https://doi.org/10.1101/2021.05.29.446277; this version posted May 31, 2021. The copyright holder for this preprint (which

was not certified by peer review) is the author/funder. This article is a US Government work. It is not subject to copyright under 17 USC 105 and is also made available for use under a CCO license.
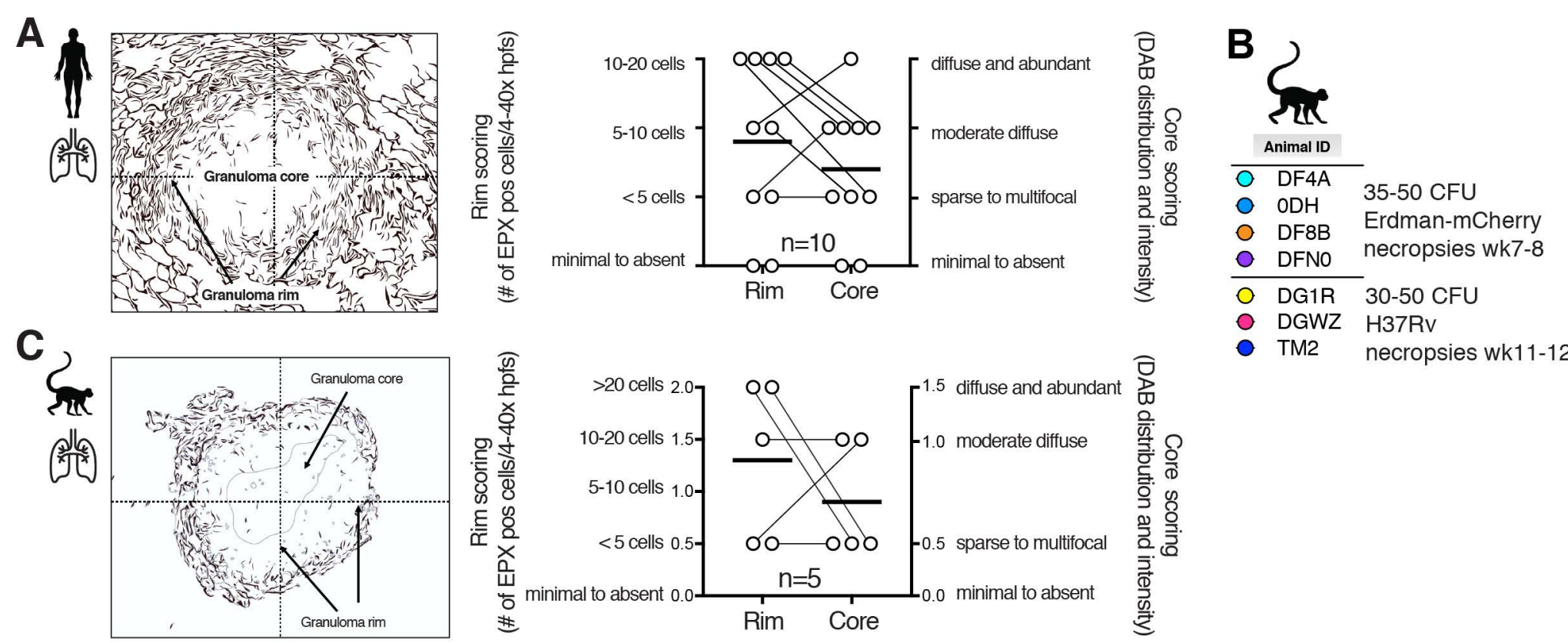

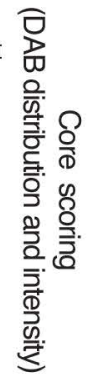

\section{D $\int_{\text {Lx }}$ 耀}

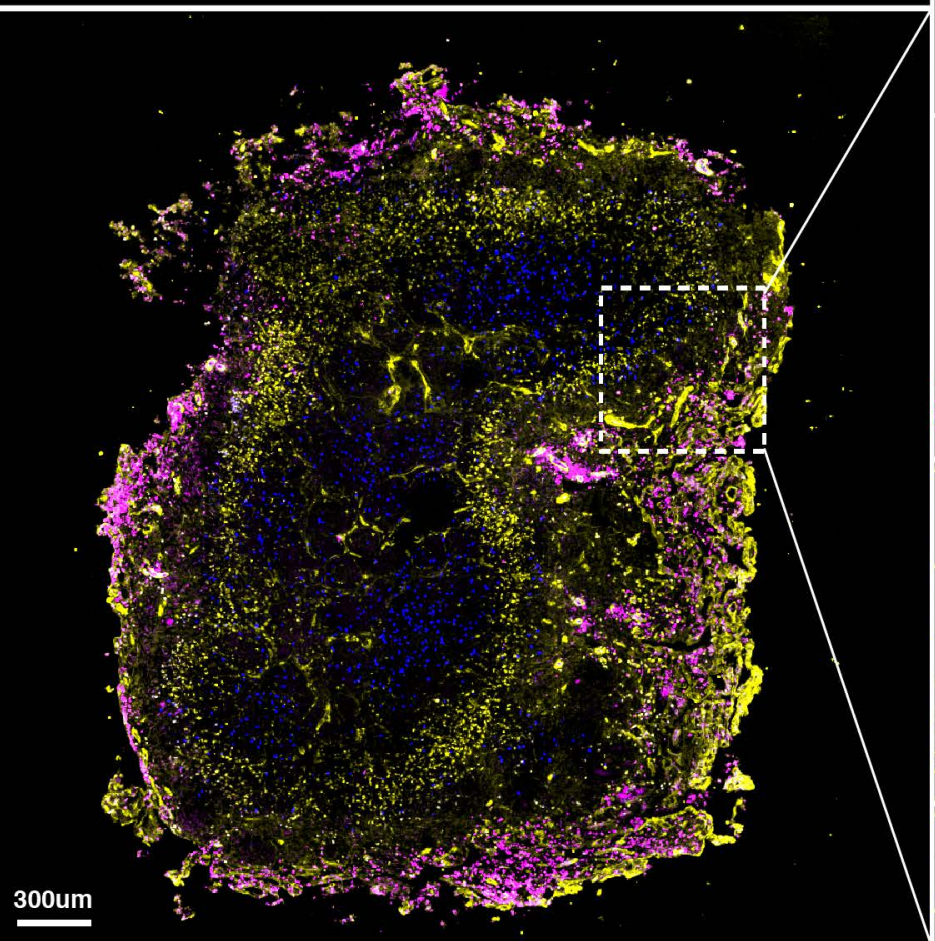

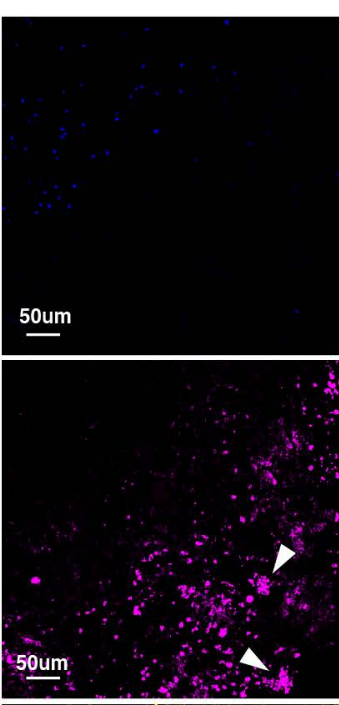
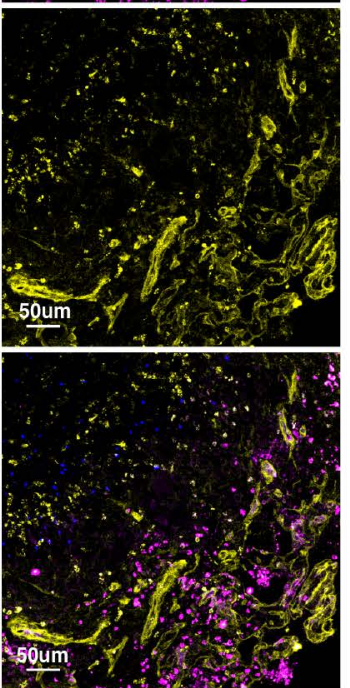

E

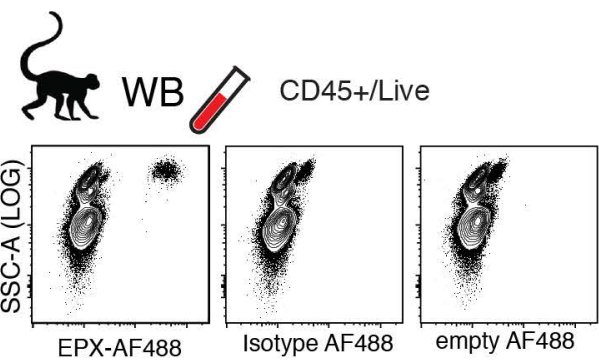

$\mathbf{F}$ Suld Granuloma O DGIR CD45+/Live/CD11 $\mathrm{b}^{+}$

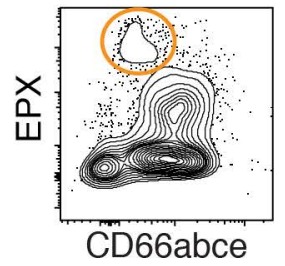



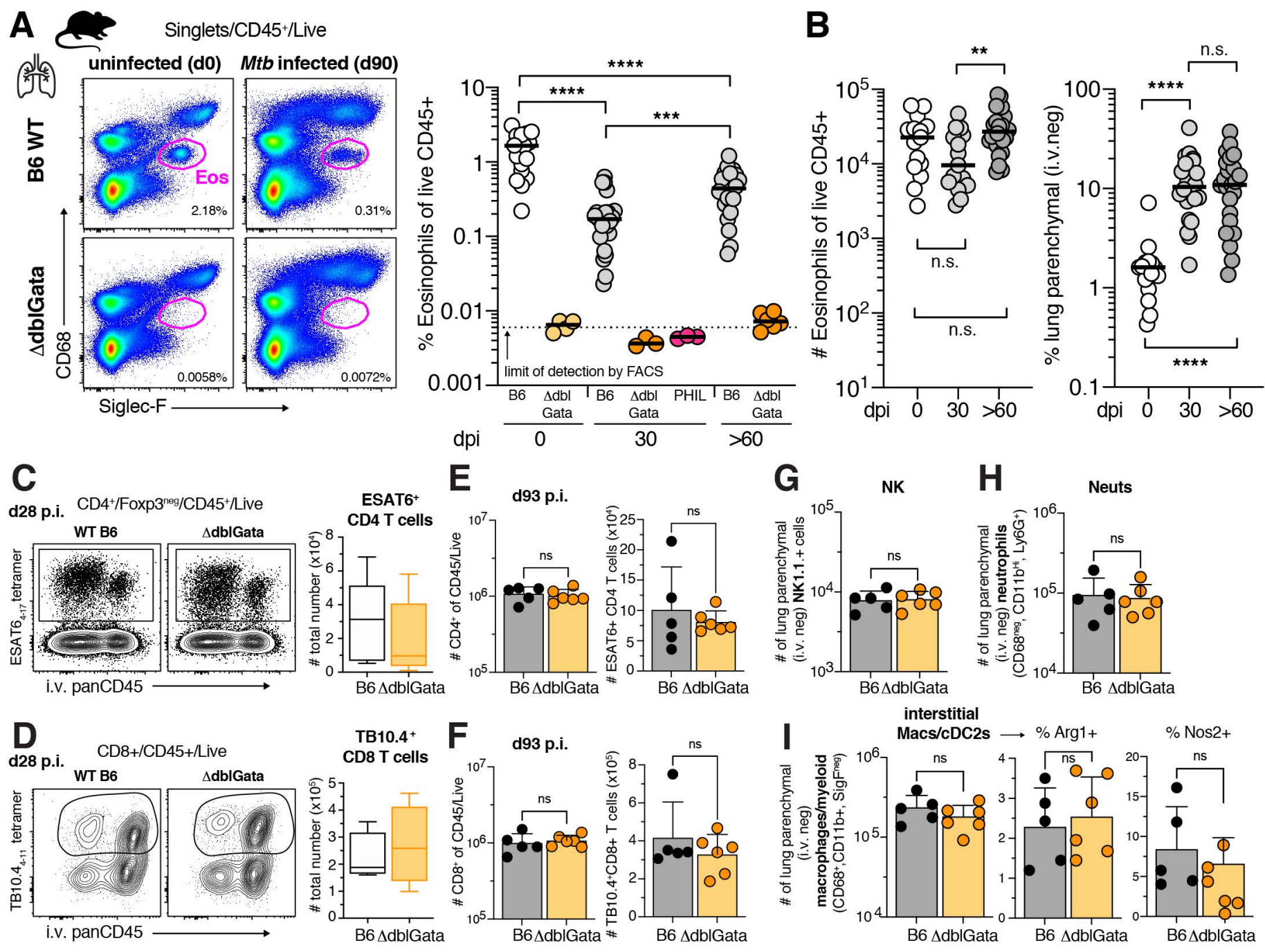

J

(CD68hi, Slglec-F+, CD11 $c^{\text {hl }}$ )

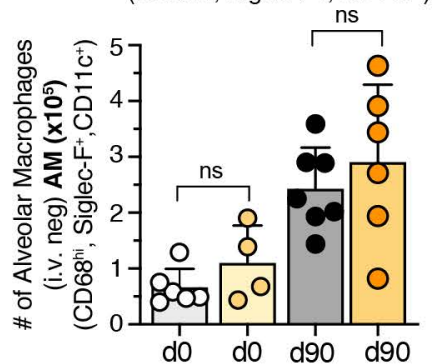

B6 $\Delta$ dblGata B6 $\Delta$ dbIGata
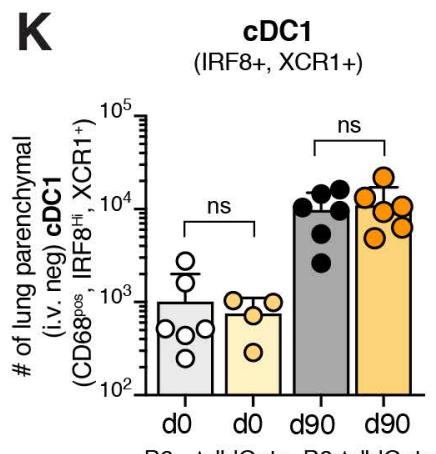

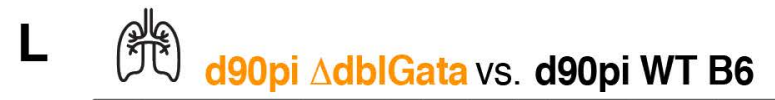

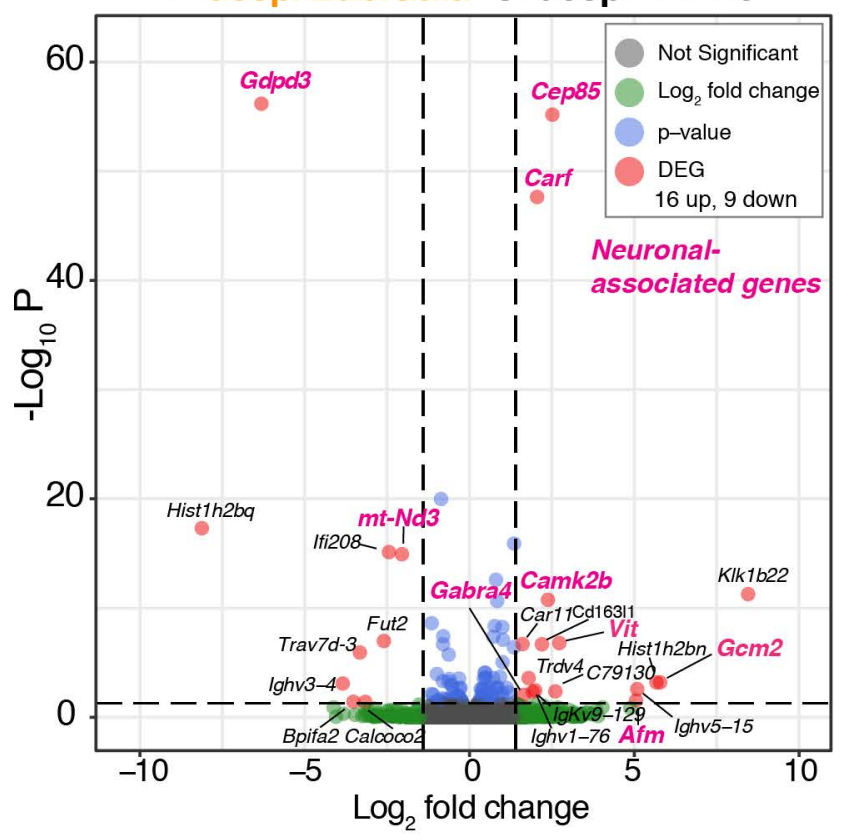

M

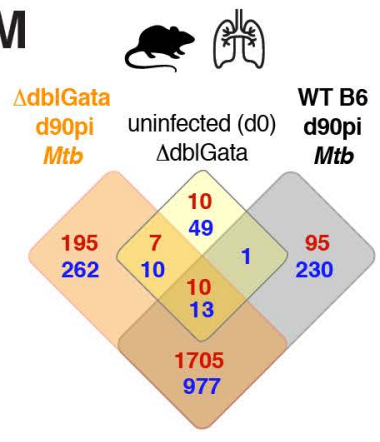

$\mathbf{N}$

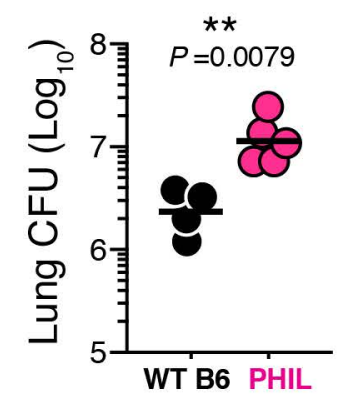

